\title{
Perturbative Renormalization of Composite Operators via Flow Equations I
}

\author{
G. Keller ${ }^{1 \star}$ and C. Kopper ${ }^{2}$ \\ 1 Max-Planck-Institut für Physik, Werner-Heisenberg-Institut, Föhringer Ring 6, \\ W-8000 München 40, FRG \\ ${ }^{2}$ Institut für Theoretische Physik, Universität Göttingen, Bunsenstrasse 9, W-3400 Göttingen, \\ FRG
}

Received November 29, 1991

\begin{abstract}
We apply the general framework of the continuous renormalization group, whose significance for perturbative quantum field theories was recognized by Polchinski, to investigate by new and mathematically simple methods the perturbative renormalization of composite operators. In this paper we demonstrate the perturbative renormalizability of the Green functions of the Euclidean massive $\Phi_{4}^{4}$ theory with one insertion of a (possibly oversubtracted, in the BPHZ language) composite operator. Moreover we show that our method admits an easy proof of the Zimmermann identities and of the Lowenstein rule.
\end{abstract}

\section{Introduction}

In the framework of the BPHZ renormalization theory the first systematic definition and study of renormalized composite operators has been performed by Zimmermann $[1,2,3]$. He introduced a special set of composite operators which can be interpreted as generalized Wick normal products and which ever since have been called Zimmermann normal products $[1,2]$. One of the remarkable features of these Zimmermann normal products is that they are a convenient tool to prove the validity of Wilson's short distance expansion in renormalized perturbation theory $[1,3]$. Moreover, Zimmermann showed $[1,2]$ that these normal products obey a set of linear relations, the Zimmermann identities, which turned out to be especially well suited for analyzing some of the main structural properties of perturbative Green functions; the importance of the Zimmermann identities can hardly be overemphasized. For instance, Lowenstein and his collaborators (mostly) have employed Zimmermann's normal product techniques and identities in conjunction with Lowenstein's rule [4] (another identity which is obeyed by the Zimmermann normal products) to construct renormalized symmetry generators (e.g. an energy momentum tensor for $\Phi_{4}^{4}$ [4]), to search for broken symmetries (e.g. asymptotic scale invariance in $\Phi_{4}^{4}[4,5,6]$ and in the 2-d massive Thirring

\footnotetext{
* Supported by the Swiss National Science Foundation
} 
model [7], or asymptotic restoration of internal symmetries $[4,5])$ and to investigate gauge invariance and (anomalous) Ward identities $[7,8]$ (e.g. also the axial vector current anomaly in QED [9]). Furthermore, similar methods lead, via the renormalized action principle [5], to a particularly elegant proof of the CallanSymanzik and the renormalization group equations [5]. A review as well as many more references on this subject can be found in $[9,10]$.

In recent years renormalization group ideas from statistical mechanics have started to influence (and in particular suggested the use of new methods in) perturbative quantum field theory. A discretized form of the renormalization group has been adapted to prove the perturbative renormalizability and local Borel summability of non-gauge theories [11] as well as of QED [12-14]. However, the renormalization of composite operators has not been carried out in this formalism.

On the other hand, also a continuous version of the renormalization group has been utilized to study problems in perturbative quantum field theory. Namely, elaborating on Polchinski's seminal work [15] we have been able to cast his continuous renormalization group or, equivalently, differential flow equation method in such a shape that a rigorous and simple proof of the perturbative renormalizability of the Euclidean massive $\Phi_{4}^{4}[16]$ as well as an extension to the Euclidean QED with a massive photon [17] has become possible. In our opinion our method exhibits only a very low degree of mathematical complexity (in particular it works without any reference to more or less involved combinatorics like Zimmermann forests or Gallavotti-Nicolò trees) but nevertheless the proofs are quite short; so we believe that it is probably preferable to BPHZ and, if one does not care about the large order bounds, also to the $\mathrm{GN}$ method $[11,12]{ }^{1}$

In this paper we continue our work on the applications of the continuous renormalization group method. We demonstrate that it can be used to control in a rather simple way also the perturbative renormalization of composite operators. One of the niceties of our approach is that an unexpectedly uncomplicated and transparent proof of the Zimmermann identities and of the Lowenstein rule emerges. In a subsequent publication [19] we will apply our method to investigate Wilson's short distance expansion.

The basic definitions and notations of our method are not standard knowledge, and the situation studied in this paper is much more general than the one in [16]. So we begin, in Sect. 2, with a detailed definition and analysis of some properties of the various quantities which later on are seen to enter the differential flow equation obeyed by the connected amputated Green functions (with none or one insertion of a composite operator) of the Euclidean massive $\Phi_{4}^{4}$; some overlap with [16] is unavoidable, but note that in contrast to [16] we do not insist on the symmetry $\phi \mapsto-\phi$. We derive the above mentioned differential flow equation and discuss the renormalization conditions which are imposed on the connected amputated Green functions (with or without one insertion).

In Sect. 3 we prove that for any renormalization conditions the Green functions of the (possibly non $\mathbb{Z}_{2}$-symmetric) Euclidean massive $\Phi_{4}^{4}$, with none or one insertion of a composite operator, are perturbatively renormalizable.

\footnotetext{
${ }^{1}$ For reasons of completeness let us also mention ref. [18] where a hybridization of the discrete and of the continuous renormalization group methods is employed to prove the perturbative renormalizability of the Euclidean massive $\Phi_{4}^{4}$. The techniques used are still more complicated than ours, and it seems as if they are also not sufficient to yield the wanted large order bounds.
} 
Section 4 is devoted to the study of Zimmermann's normal products. Using the fact that the differential flow equation for the Green functions with one insertion is linear we readily realize that very general linear combinations of Green functions with one insertion of arbitrary composite operators also obey the same (linear) differential flow equation. It is trivial to check that a solution of this linear differential flow equation vanishes if the boundary conditions are zero. Now, the nontrivial boundary conditions on the Green functions with one insertion are determined by the renormalization conditions. Combining this we arrive without too much effort at our main conclusion (Theorem 8) that for quite general renormalization conditions on the Green functions with one insertion there exist linear relations among composite operators of equal or unequal dimensions. This result might be called a generalized Zimmermann identity. In particular, it holds true if we impose those renormalization conditions which define Zimmermann's normal products, $N^{p}[\cdots]$, where the index " $p$ " is not at all standard but is useful for our purposes. Now, the $N^{p}[\cdots]$ are defined by renormalization conditions which in principle look natural but which are imposed on the proper functions containing one insertion of the corresponding composite operator (thus our index " $p$ "). This is natural for BPHZ but not for us, because all we ever need to "see" in our approach are the connected Green functions, i.e. we do not have to care about the substructure of the connected Feynman diagrams. So for us it is more natural to define the new Zimmermann normal products $N^{c}[\cdots]$, defined by the same natural renormalization conditions but imposed on the connected amputated Green functions with one insertion. Theorem 8 applies to the $N^{c}[\cdots]$ as well, and it is easy to compute the coefficients in the Zimmermann identities obeyed by the $N^{c}[\cdots]$. Moreover Theorem 8 can be used to show that $N^{c}[\cdots]$ may be written as a (in general nontrivial) linear combination of the standard normal products $N^{p}[\cdots]$. We then apply the same simple scheme (i.e. $f$ solves the linear differential flow equation $+f$ has zero boundary conditions $\Rightarrow f \equiv 0$ ) once more to prove the Lowenstein rule and the $O(4)$ covariant transformation property for the $N^{c}[\cdots]$. As far as we can see the normal products $N^{c}[\cdots]$, which from our point of view are more natural, are just as well suited for applications as Zimmermann's original $N^{p}[\cdots]$.

\section{Flow Equations for Green Functions}

2.1. Let $\Lambda_{0}, 0<\Lambda_{0}<\infty$, be a momentum space cutoff and $\Lambda \in\left[0, \Lambda_{0}\right]$ a scale parameter. We take $m^{2}>0$ and define, for $x, y \in \mathbb{R}^{4}$, the regularized free Euclidean propagator, $C_{\Lambda}^{\Lambda_{0}}$, by

$$
C_{\Lambda}^{\Lambda_{0}}(x, y):=\int \frac{d^{4} p}{(2 \pi)^{4}} \frac{e^{i p(x-y)}}{p^{2}+m^{2}}\left(R\left(\Lambda_{0}, p\right)-R(\Lambda, p)\right) .
$$

Here $R(\Lambda, p)$ is a momentum space regularizing function which is chosen to have the form

$$
R(\Lambda, p):=\left(1-e^{-(\Lambda / m)^{\theta}}\right) \cdot K\left(\frac{p^{2}}{\Lambda^{2}}\right),
$$

where $\theta$ is an arbitrary but fixed real number obeying $\theta \geqq \theta_{\min }, \theta_{\min } \geqq 4$ to be specified later on, and where $K \in C^{\infty}[0, \infty)$ satisfies $0 \leqq K \leqq 1, K(a)=1$ for $0 \leqq a \leqq 1, K(a)=0$ for $4 \leqq a$. 
In fact it is irrelevant for our purposes which $\theta$ is chosen, as long as $\theta \geqq \theta_{\min }$, because we prove in the Appendix that in the limit $\Lambda_{0} \rightarrow \infty$ the renormalized Green functions regularized with $\theta$ coincide with those regularized with $\theta^{\prime}$, if $\theta, \theta^{\prime} \geqq \theta_{\min }$. Note that $R(0, p) \equiv 0$, so $R(\Lambda, p) \in C^{\infty}\left(\mathbb{R}^{4}\right)$ for all $\Lambda \in[0, \infty)$, and $\theta$ has the effect that $R(\Lambda, p) \in C^{(\theta-1)}\left([0, \infty) \times \mathbb{R}^{4}\right)$; obviously $R(\Lambda, p) \in C^{\infty}\left((0, \infty) \times \mathbb{R}^{4}\right)$. Similar smoothness properties hold for $C_{\Lambda}^{\Lambda_{0}}(x, y)$; moreover $C_{\Lambda}^{\Lambda_{0}}(x, y)=\left(C_{\Lambda}^{\Lambda_{0}}(x, y)\right)^{*}=$ $C_{\Lambda}^{\Lambda_{0}}(y, x)=C_{\Lambda}^{\Lambda_{0}}(x-y)$. Observe that, roughly speaking, $C_{\Lambda}^{\Lambda_{0}}$ is obtained by integrating out all the momenta $p$ with $|p| \in\left[\Lambda, \Lambda_{0}\right]$.

Assume that $\phi \in \mathscr{S}\left(\mathbb{R}^{4}\right)$ and introduce the notation $\delta_{\phi}(x):=\delta / \delta \phi(x),\left\langle f_{1}, f_{2}\right\rangle$ $:=\int d^{4} x f_{1}(x) f_{2}(x)$. We define the functional Laplace operator, $\Delta\left(\Lambda, \Lambda_{0}\right)$, by the formula

$$
\Delta\left(\Lambda, \Lambda_{0}\right):=\frac{1}{2}\left\langle\delta_{\phi}, C_{\Lambda}^{\Lambda_{0}} \delta_{\phi}\right\rangle
$$

2.2. We introduce formal variables $g_{1}, g_{2}$ and $\lambda$, an intermediate volume cutoff $V$, i.e. a finite volume $V \subset \mathbb{R}^{4}$, and a source function $\chi, \chi \in \mathscr{S}\left(\mathbb{R}^{4}\right)$. Then the effective Lagrangian at scale $\Lambda, L^{\Lambda, \Lambda_{0} ; V}$, is defined as follows.

a) We demand that $L^{\Lambda, \Lambda_{0} ; V}$ is a formal power series (fps) in $g_{1}, g_{2}$ and $\lambda$, thus

$$
L^{\Lambda, \Lambda_{0} ; V}:=\sum_{|r| \geqq 0, t \geqq 0} g^{r} \lambda^{t} L_{r, t}^{\Lambda, \Lambda_{0} ; V},
$$

where $r \equiv\left(r_{1}, r_{2}\right), r_{1}, r_{2} \in \mathbb{N}_{0}, g^{r} \equiv g_{1}^{r_{1}} g_{2}^{r_{2}},|r| \equiv r_{1}+r_{2}$.

b) For $\Lambda=\Lambda_{0}$ we require that

$$
L^{\Lambda_{0}, \Lambda_{0} ; V}:=l^{\Lambda_{0} ; V}-\lambda \cdot \int_{V} d^{4} x \chi(x) \cdot B_{D}(x),
$$

where $l^{\Lambda_{0}} ; V$ represents the bare interaction for the possibly $\mathbb{Z}_{2}$-nonsymmetric but Euclidean invariant $\Phi_{4}^{4}$ theory:

$$
\begin{aligned}
& l^{\Lambda_{0} ; V}=\sum_{|r| \geqq 0} g^{r} l_{r}^{\Lambda_{0} ; V} \\
& l_{r}^{\Lambda_{0} ; V}:=\left\{\begin{array}{l}
0, \quad|r|=0 \\
\int_{V} d^{4} x\left[a_{r}^{(1)} \phi(x)+a_{r}^{(2)} \phi^{2}(x)+a_{r}^{(3)} \phi \square \phi+a_{r}^{(4)} \phi^{3}+a_{r}^{(5)} \phi^{4}\right], \quad|r| \geqq 1 .
\end{array}\right.
\end{aligned}
$$

The coefficients $a_{r}^{(j)}, 1 \leqq j \leqq 5$, will become uniquely determined functions of $\Lambda_{0}$, $m^{2}, R, r$ and of the renormalization conditions (on the $\Phi_{4}^{4}$ Green functions without operator insertions) once the latter are specified. For instance, if we wish to interpret $g_{1}, g_{2}$ as the "coupling constants" of the $\phi^{3}$ and $\phi^{4}$ interaction vertices, respectively, we will impose renormalization conditions such that at least $a_{(1,0)}^{(4)}=\frac{1}{3 !}, a_{(0,1)}^{(5)}=\frac{1}{4 !}, a_{(0,1)}^{(4)}=a_{(1,0)}^{(5)}=0$.

Next, the second term on the r.h.s. of (2.5) is meant to generate insertions of the local polynomial $B_{D}(x)$, the index $D$ indicating that $B_{D}$ is of dimension $\leqq D$ in the field $\phi$ and its derivatives. Thus we impose that $D \geqq 1$ and that

$$
B_{D}(x):=b_{0}+\sum_{n=1}^{D} \sum_{\{w\}:|w|+n \leqq D} b_{n,\{w\}} \partial_{x}^{w_{1}} \phi(x) \ldots \partial_{x}^{w_{n}} \phi(x),
$$


where $\{w\}:=\left\{w_{1}, \ldots, w_{n}\right\},|w|:=\sum_{i=1}^{n}\left|w_{i}\right|$, and for $j \in\{1, \ldots, n\}$ we have $w_{j}:=\left(w_{j, 1}, \ldots, w_{j, 4}\right),\left|w_{j}\right|:=\sum_{\mu=1}^{4} w_{j, \mu}, w_{j, \mu} \in \mathbb{N}_{0}$ for $1 \leqq \mu \leqq 4$, and finally $\partial_{x}^{w_{j}}:=\prod_{\mu=1}^{4} \partial^{w_{J, \mu}} /\left(\partial x_{\mu}\right)^{w_{j, \mu}}$; and $b_{0}, b_{n,\{w\}}$ are fps in $g_{1}, g_{2}$, i.e.

$$
b_{0}=\sum_{|r| \geqq 0} g^{r} b_{r ; 0}, \quad b_{n,\{w\}}=\sum_{|r| \geqq 0} g^{r} b_{r ; n,\{w\}} .
$$

Here the coefficients $b_{r ; 0}$ and $b_{r ; n,\{w\}}$ will be seen to be uniquely determined functions of $\Lambda_{0}, m^{2}, R, r,\left\{a_{r^{\prime}}^{(j)}\right\}$ and of the renormalization conditions (on the Green functions with one insertion of $B_{D}$ ) once these latter are specified. In general $B_{D}(x)$ will not be Euclidean invariant.

c) For $\Lambda \in\left[0, \Lambda_{0}\right]$ we define the fps $S^{\Lambda, \Lambda_{0} ; V}$ by the flow equation

$$
e^{-S^{\Lambda, \Lambda_{0}: V}}:=e^{\Delta\left(\Lambda, \Lambda_{0}\right)} e^{-L^{\Lambda_{0}, A_{0}, V}} .
$$

Expanding both sides of (2.9) as fps we first note that (2.9) does not fix uniquely the coefficient $S_{(0,0), 0}^{\Lambda, \Lambda_{0} ; V}$ because the only condition which it has to respect is $e^{-S_{(0,0 ;,}^{A}, A_{0}, V}=1$. However, since (2.9) tells us that for $|r|+t \geqq 1$ the coefficients $S_{r, t}^{\Lambda, \Lambda_{0} ; V}$ are independent of $S_{(0,0), 0}^{\Lambda, \Lambda_{0} ; V}$ (so in particular they are unaffected by the

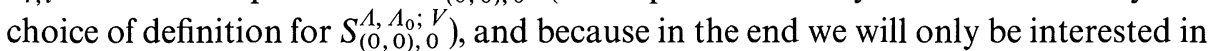
$\left\{S_{r, t}^{\Lambda, \Lambda_{0} ; V}:|r|+t \geqq 1\right\}$ we choose now for convenience

$$
S_{(0,0), 0}^{\Lambda, \Lambda_{0} ; V}:=0 \text {. }
$$

Second, the coefficients $S_{r, t}^{\Lambda, \Lambda_{0}} ; V,|r|+t \geqq 1$, can by induction be seen to be polynomials in $\phi$ and $\chi$ which are uniquely determined; they are finite for all $\Lambda \in\left[0, \Lambda_{0}\right]$ and $V<\infty$. Moreover they are endowed with all the smoothness properties which are naively expected to hold, e.g. w.r.t. $\Lambda$ they belong to $C^{(\theta-1)}\left[0, \Lambda_{0}\right]$ and $C^{\infty}\left(0, \Lambda_{0}\right]$. Due to $(2.10)$ the $S_{r, t}^{\Lambda, \Lambda_{0}} ; V,|r|+t \geqq 0$, are polynomials in $\phi$ and $\chi$ and so we may define $I^{\Lambda, \Lambda_{0}} ; V$ to be the fps which comprises precisely the field independent parts of $S^{\Lambda, \Lambda_{0} ; V}$ (where both $\phi$ and $\chi$ are called fields). Using (2.9), (2.10) we observe that $S^{\Lambda, \Lambda_{0} ; V}-I^{\Lambda, \Lambda_{0} ; V}$ exhibits the same smoothness properties as $S^{\Lambda, \Lambda_{0} ; V}$. Because (2.9), (2.10) also imply that $S^{\Lambda_{0}, \Lambda_{0} ; V}=L^{\Lambda_{0}, \Lambda_{0} ; V}$ and since $L^{\Lambda_{0}, \Lambda_{0} ; V}$ contains no field independent contributions (cf. (2.5), (2.6)) we see that $I^{\Lambda_{0}}, \Lambda_{0} ; V=0$; in other words $L^{\Lambda_{0}, \Lambda_{0} ; V}=S^{\Lambda_{0}, \Lambda_{0} ; V}-I^{\Lambda_{0}, \Lambda_{0} ; V}$. Therefore it makes sense to define, for $\Lambda \in\left[0, \Lambda_{0}\right)$,

$$
L^{\Lambda, \Lambda_{0} ; V}:=S^{\Lambda, \Lambda_{0} ; V}-I^{\Lambda, \Lambda_{0} ; V} .
$$

2.3. The effective Lagrangian $L^{\Lambda, \Lambda_{0} ; V}$ is the generating functional for the connected Green functions (at perturbative order $|r|+t \geqq 1$ ), and this fact can be proven as follows.

Proposition 1. (At least) for $\phi, \chi, J \in \mathscr{S}\left(\mathbb{R}^{4}\right)$ we find that

$$
\left.e^{\Delta\left(\Lambda, \Lambda_{0}\right)} e^{-L^{\Lambda_{0}, A_{0} ; V}(\phi, \chi)}\right|_{\phi=C_{\Lambda}^{A_{0} J}}=e^{-1 / 2\left\langle J, C_{\Lambda}^{\left.A_{0} J\right\rangle}\right.} e^{-L^{\Lambda_{0}, \Lambda_{0} ;}\left(\delta_{J}, \chi\right)} e^{1 / 2\left\langle J, C_{A}^{\left.A_{0} J\right\rangle} .\right.}
$$

Proof. The proof is a combinatoric exercise which perhaps is best carried out by expanding (2.12) in powers of $L^{\Lambda_{0}, \Lambda_{0} ; V}$ and by verifying that (2.12) holds at each power in $L^{\Lambda_{0}, \Lambda_{0} ; V}$ separately. For the purpose of proving (2.12) it is sufficient to assume that $L_{r, t}^{\Lambda_{0}, \Lambda_{0} ; V}$ is a monomial in $\phi$ (and $\chi$ ); so the proof of (2.12) is reduced to proving that (2.12) is true if we replace $e^{-L^{\Lambda_{0}, \Lambda_{0}: V}}$ by a monomial in $\phi$ (and $\chi$ ) which 
is not difficult if we use induction in the degree of this monomial and remember that $C_{\Lambda}^{\Lambda_{0}}(x, y)=C_{\Lambda}^{\Lambda_{0}}(y, x)$. More details can be found in ref. [16], Lemma 2.

The generating functional, $Z^{\Lambda, \Lambda_{0} ; V}(J, \chi)$, of the unamputated and unnormalised Green functions of the Euclidean quantum field theory built from the free propagator $C_{\Lambda}^{\Lambda_{0}}$ and from the interaction vertices $L^{\Lambda_{0}, \Lambda_{0} ; V}$ is given by

$$
\begin{aligned}
Z^{\Lambda, \Lambda_{0} ; V}(J, \chi) & =\int d \mu_{C_{\Lambda}^{\Lambda_{0}}}(\varphi) e^{-L^{\Lambda_{0}, \Lambda_{0}, V}(\varphi, \chi)+\langle J, \varphi\rangle} \\
& =e^{-L^{\Lambda_{0}, \Lambda_{0} ;} V_{\left(\delta_{J}, \chi\right)}} e^{1 / 2\left\langle J, C_{A}^{\left.\Lambda_{0} J\right\rangle},\right.}
\end{aligned}
$$

where $d \mu_{C_{\Lambda}^{\Lambda_{0}}}$ is the Gaussian measure with mean zero and covariance $C_{\Lambda}^{\Lambda_{0}}$. Combining (2.13), (2.12) and (2.9), (2.11) we see that (modulo an irrelevant $2 \pi i \mathbb{Z}$ )

$$
\log Z^{\Lambda, \Lambda_{0} ; V}(J, \chi)=\frac{1}{2}\left\langle J, C_{\Lambda}^{\Lambda_{0}} J\right\rangle-L^{\Lambda, \Lambda_{0} ; V}(\phi, \chi)-\left.I^{\Lambda, \Lambda_{0} ; V}\right|_{\phi=C_{\Lambda}^{\Lambda_{0} J}} .
$$

Since $\left\langle J, C_{\Lambda}^{\Lambda_{0}} J\right\rangle$ is $0^{\text {th }}$ order in $g, \lambda$ and $I^{\Lambda, \Lambda_{0} ; V}$ contains no fields we arrive at the

Proposition 2. $L^{\Lambda, \Lambda_{0} ; V}(\phi, \chi)$ is the generating functional of the order $|r|+t \geqq 1$ perturbative connected amputated Green functions of the Euclidean quantum field theory defined by the propagator $C_{\Lambda}^{\Lambda_{0}}$ and the vertices $L^{\Lambda_{0}, \Lambda_{0} ; V}$.

In retrospect this result shows that the definition (2.10) has also been sensible (apart from being convenient), because due to $(2.10)$ the $0^{\text {th }}$ order contribution to $L^{\Lambda, \Lambda_{0} ; V}$ vanishes implying that $-\frac{1}{2}\left\langle J, C_{\Lambda}^{\Lambda_{0}} J\right\rangle$ alone generates the $0^{\text {th }}$ order connected Green functions, as it should be.

As a Corollary to Proposition 2 we observe that for all $\Lambda \in\left[0, \Lambda_{0}\right]$ the limit

$$
L^{\Lambda, \Lambda_{0}}:=\lim _{V \uparrow \mathbb{R}^{4}} L^{\Lambda, \Lambda_{0} ; V}
$$

exists, because $L^{\Lambda, \Lambda_{0} ; V}$ is a sum over connected Feynman diagrams each of which possesses at least one external field $\left(\phi\right.$ or $\chi$ ) and because the propagator $C_{\Lambda}^{\Lambda_{0}}$ has fast decay in $x$-space.

Set $\partial_{\Lambda}:=\partial / \partial \Lambda$. With this notation the definitions (2.9), (2.10) and (2.3) imply that, for $\Lambda \in\left[0, \Lambda_{0}\right], S^{\Lambda, \Lambda_{0} ; V}$ obeys the functional differential equation

$$
\partial_{\Lambda} S^{\Lambda, \Lambda_{0} ; V}=\left(\partial_{\Lambda} \Delta\left(\Lambda, \Lambda_{0}\right)\right) S^{\Lambda, \Lambda_{0} ; V}-\frac{1}{2}\left\langle\delta_{\phi} S^{\Lambda, \Lambda_{0} ; V},\left(\partial_{\Lambda} C_{\Lambda}^{\Lambda_{0}}\right) \delta_{\phi} S^{\Lambda, \Lambda_{0} ; V}\right\rangle .
$$

Due to (2.11) we obtain the differential flow equation for $L^{\Lambda, \Lambda_{0}}$ :

$$
\partial_{\Lambda} L^{\Lambda, \Lambda_{0}}=\left.\left(\partial_{\Lambda} \Delta\left(\Lambda, \Lambda_{0}\right)\right) L^{\Lambda, \Lambda_{0}}\right|_{\text {field-dep. }}-\left.\frac{1}{2}\left\langle\delta_{\phi} L^{\Lambda, \Lambda_{0}},\left(\partial_{\Lambda} C_{\Lambda}^{\Lambda_{0}}\right) \delta_{\phi} L^{\Lambda, \Lambda_{0}}\right\rangle\right|_{\text {field-dep. }} .
$$

2.4. We expand $L^{\Lambda, \Lambda_{0}}$ as a fps in $g_{1}, g_{2}$ and $\lambda$

$$
L^{\Lambda, \Lambda_{0}}=\sum_{|r| \geqq 0, t \geqq 0} g^{r} \lambda^{t} L_{r, t}^{\Lambda, \Lambda_{0}} .
$$


From Proposition 2 we know that $L_{r}^{\Lambda, 0}, \Lambda_{0}$ and $L_{r, 1}^{\Lambda, \Lambda_{0}}$ have the following momentum space representation and interpretation:

$\underline{t=0}: \quad L_{r, 0}^{\Lambda, \Lambda_{0}}=\mathscr{L}_{r, 0 ; 1}^{\Lambda, \Lambda_{0}} \cdot \int d^{4} x \phi(x)$

$$
+\sum_{n=2}^{\infty} \int \prod_{j=1}^{n-1} \frac{d^{4} p_{j}}{(2 \pi)^{4}} \hat{\phi}\left(p_{1}\right) \ldots \hat{\phi}\left(p_{n}\right) \cdot \mathscr{L}_{r, 0 ; n}^{\Lambda, \Lambda_{0}}\left(p_{1}, \ldots, p_{n-1}\right),
$$

where

a) $p_{n}:=-\sum_{j=1}^{n-1} p_{j}$

b) we may assume without loss of generality that, for $n \geqq 2, \mathscr{L}_{r, 0 ; n}^{\Lambda, \Lambda_{0}}\left(p_{1}, \ldots, p_{n-1}\right)$ $=\mathscr{L}_{r, 0 ; n}^{\Lambda, \Lambda_{0}}\left(p_{\pi(1)}, \ldots, p_{\pi(n-1)}\right), \forall \pi \in S_{n}$;

c) the sum over $n$ in (2.18) actually extends only over finitely many $n$ because, as we noted earlier, $L_{r, t}^{\Lambda, \Lambda_{0}}$ is a polynomial in $\phi$ and $\chi$; in particular, since just the connected $\Phi^{4}$-diagrams contribute to $\mathscr{L}_{r, 0 ; n}^{\Lambda, \Lambda_{0}}$ we easily see that

$$
\mathscr{L}_{r, 0 ; n}^{\Lambda, \Lambda_{0}} \equiv 0, \quad \text { if } n>2|r|+2 ;
$$

d) for $|r| \geqq 1 \mathscr{L}_{r, 0 ; n}^{\Lambda=0, \Lambda_{0}}$ is the connected amputated $n$-point Green function of the $\Phi_{4}^{4}$ theory, with no insertions of $B_{D}$, at perturbative order $r$ and with UV-cutoff $\Lambda_{0}$; for $|r|=0$ we have $\mathscr{L}_{r, 0 ; n}^{\Lambda, \Lambda_{0}} \equiv 0$;

e) $\mathscr{L}_{r, 0 ; n}^{\Lambda, \Lambda_{0}} \in C^{(\theta-1)}\left(\left[0, \Lambda_{0}\right] \times \mathbb{R}^{4(n-1)}\right) ; \mathscr{L}_{r, 0 ; n}^{\Lambda, \Lambda_{0}} \in C^{\infty}\left(\left(0, \Lambda_{0}\right] \times \mathbb{R}^{4(n-1)}\right)$ and is invariant under the orthogonal group; this latter property implies that for all $\Lambda$

$$
\left.\partial_{p}^{w} \mathscr{L}_{r, 0 ; n}^{\Lambda, \Lambda_{0}}\left(p_{1}, \ldots, p_{n-1}\right)\right|_{p_{1}=\cdots=p_{n-1}=0}=0, \text { if }|w|=1,
$$

where $w$ is the multiindex $w=\left(w_{1}, \ldots, w_{n-1}\right), \partial_{p}^{w} \equiv \partial_{p_{1}}^{w_{1}} \ldots \partial_{p_{n-1}}^{w_{n-1}}$, and for $n=2$ e.g. we have the proportionality

$$
\left.\partial_{p_{\mu}} \partial_{p_{v}} \mathscr{L}_{r, 0 ; 2}^{\Lambda, \Lambda_{0}}(p)\right|_{p=0} \sim \delta_{\mu, v} .
$$

$\underline{t=1}: \quad L_{r, 1}^{\Lambda, \Lambda_{0}}=\mathscr{L}_{r, 1 ; 0}^{\Lambda, \Lambda_{0}} \cdot \int d^{4} x \chi(x)$

$$
+\sum_{n=1}^{\infty} \int \prod_{j=1}^{n} \frac{d^{4} p_{j}}{(2 \pi)^{4}} \hat{\phi}\left(p_{1}\right) \ldots \hat{\phi}\left(p_{n}\right) \cdot \hat{\chi}\left(p_{n+1}\right) \cdot \mathscr{L}_{r, 1 ; n}^{\Lambda, \Lambda_{0}}\left(p_{1}, \ldots, p_{n}\right),
$$

where remarks similar to a)-d) before apply to $\mathscr{L}_{r, 1 ; n}^{\Lambda, \Lambda_{0}}$. In particular $\mathscr{L}_{r, 1 ; n}^{0, \Lambda_{0}}$ is the connected amputated $n$-point Green function of the $\Phi_{4}^{4}$ theory with 1 insertion of $B_{D}(x)$ at $x=0$, at perturbative order $r,|r| \geqq 0$, and with UV-cutoff $\Lambda_{0}$. In general $\mathscr{L}_{r, 1 ; n}^{\Lambda, \Lambda_{0}}$ will not be invariant under the orthogonal group.

We insert the expansions (2.18), (2.22) into (2.16), and after some easy calculations we arrive at the differential flow equations for $\left\{\mathscr{L}_{r, t ; n}^{\Lambda, \Lambda_{0}}: t=0,1\right\}$ :

$$
\begin{aligned}
\partial_{\Lambda} \mathscr{L}_{r, t ; n}^{\Lambda, \Lambda_{0}}\left(p_{1}, \ldots, p_{n+t-1}\right)= & -\left(\begin{array}{c}
n+2 \\
2
\end{array}\right) \int \frac{d^{4} q}{(2 \pi)^{4}} \frac{\partial_{\Lambda} R(\Lambda, q)}{q^{2}+m^{2}} \\
& \cdot \mathscr{L}_{r, t ; n+2}^{\Lambda, \Lambda_{0}}\left(q,-q, p_{1}, \ldots, p_{n+t-1}\right) \\
& +\sum_{r^{\prime}+r^{\prime \prime}=r}(n+1) \cdot \frac{\partial_{\Lambda} R(\Lambda, 0)}{m^{2}} \cdot \mathscr{L}_{r^{\prime}, 0 ; 1}^{\Lambda, \Lambda_{0}} \\
& \cdot \mathscr{L}_{r^{\prime \prime}, t ; n+1}^{\Lambda, \Lambda_{0}}\left(0, p_{1}, \ldots, p_{n+t-1}\right)
\end{aligned}
$$




$$
\begin{aligned}
& +\sum_{\substack{r^{\prime}+r^{\prime \prime}=r \\
n^{\prime}+n^{\prime \prime}=n+2}}^{*} n^{\prime} n^{\prime \prime} 2^{t-1} \cdot\left[\frac{\partial_{\Lambda} R(\Lambda, Q)}{Q^{2}+m^{2}}\right. \\
& \cdot \mathscr{L}_{r^{\prime}, 0 ; n^{\prime}}^{\Lambda, \Lambda_{0}}\left(p_{1}, \ldots, p_{n^{\prime}-1}\right) \\
& \left.\cdot \mathscr{L}_{r^{\prime \prime}, t ; n^{\prime \prime}}^{\Lambda, \Lambda_{0}}\left(-Q, p_{n^{\prime}}, \ldots, p_{n+t-1}\right)\right]_{\mathrm{symm}},
\end{aligned}
$$

where $\sum^{*}$ indicates that the sum extends over $n^{\#} \geqq 2$, if $t=0$, and over $n^{\prime} \geqq 2$, $n^{\prime \prime} \geqq 1$, if $t=1$; moreover $Q:=-\sum_{j=1}^{n^{\prime}-1} p_{j}$, and $[\cdots]_{\text {symm }}$ means symmetrization w.r.t. the momenta $p_{1}, \ldots, p_{n}$. Although it may seem at first sight that the differential equation (2.23) holds only for those $\mathscr{L}_{r, t ; n}^{\Lambda, \Lambda_{0}}$ with $n+t-1 \geqq 1$, it in fact remains valid if $n+t-1=0$; obviously in this latter case the second sum on the r.h.s. of (2.23) is absent.

2.5. On the space of sufficiently regular functions $f: \mathbb{R}^{4 n} \rightarrow \mathbb{C}$ we introduce a set of norms, \|\|$_{(a, b)}$, where $a, b \in \mathbb{R}_{+}$. For instance we define that for $z \in \mathbb{N}_{0}$

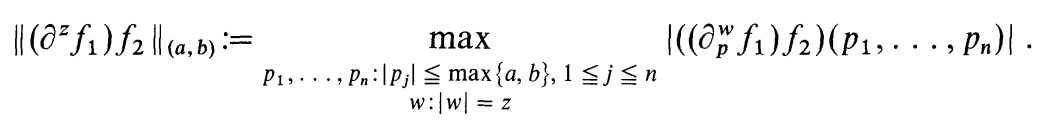

Next, we choose for technical reasons a scale, $\Lambda_{1}$, which is supposed to obey $0<\Lambda_{1}<\Lambda_{0}$ and to be independent of $\Lambda_{0} ; \Lambda_{1}$ is fixed once and for all. For example, this can be accomplished by requiring (without loss of generality) that $\Lambda_{0} \geqq \Lambda_{0, \min }>0$ and that $\Lambda_{1}:=\frac{1}{2} \Lambda_{0, \min }$. Put

$$
f_{\beta}(p):=\left(p^{2}+m^{2}\right)^{\beta}, \quad \beta \geqq 0 .
$$

Using (2.2) and (2.24), (2.25) it is not difficult to check that

$$
\left\|\left(\partial^{z} \partial_{\Lambda} R(\Lambda, \cdot)\right) \cdot f_{\beta}^{-1}\right\|_{(1, \infty)} \leqq \begin{cases}c \cdot \Lambda^{\theta-1-z}, & 0 \leqq \Lambda \leqq \Lambda_{1} \\ c^{\prime} \cdot \Lambda^{-2 \beta-1-z}, & \Lambda_{1} \leqq \Lambda \leqq \Lambda_{0}\end{cases}
$$

where $c, c^{\prime}$ do not depend on $\Lambda$, but of course $c, c^{\prime}$ depend on $z, R, m^{2}, \Lambda_{1}, \beta$.

Acting with $\partial_{p}^{w}$ on the differential flow equation (2.23) and estimating it with the help of (2.26) we find that for $t=0,1$ and $\eta \geqq 0, \eta$ arbitrary, and $\Lambda \in\left[0, \Lambda_{1}\right]$, $z \leqq \theta-1$ :

$$
\begin{aligned}
\left\|\partial_{\Lambda} \partial^{z} \mathscr{L}_{r, t ; n}^{\Lambda, \Lambda_{0}}\right\|_{(2 \Lambda, \eta)} \leqq & \text { const } \\
& +\sum_{r^{\prime}+r^{\prime \prime}=r}\left\|\partial^{z} \mathscr{L}_{r, t ; n+2}^{\Lambda, \Lambda_{0}}\right\|_{(2 \Lambda, \eta)} \\
+ & \sum_{\substack{r^{\prime}+r^{\prime \prime}=r \\
n^{\prime}+n^{\prime \prime}=n+1 \\
z^{\prime}+z^{\prime \prime} \leqq z}}^{\Lambda, \Lambda_{0}}\left\|_{(2 \Lambda, \eta)} \cdot\right\| \partial^{z} \mathscr{L}_{r^{\prime \prime}, t ; n+1}^{\Lambda, \Lambda_{0}} \|_{(2 \Lambda, \eta)} \\
& \left(0 \leqq \Lambda \leqq \partial^{z^{\prime}} \mathscr{L}_{r^{\prime}, 0 ; n^{\prime}}^{\Lambda, \Lambda_{0}}\left\|_{(2 \Lambda, \eta)} \cdot\right\| \partial^{z^{\prime \prime}} \mathscr{L}_{r^{\prime \prime}, t ; n^{\prime \prime}}^{\Lambda, \Lambda_{0}} \|_{(2 \Lambda, \eta)}\right\}
\end{aligned}
$$

where "const" stands for some number which depends neither on $\Lambda$ non $\Lambda_{0}$ nor on $\eta$. Of course $\left\|\partial^{z} \mathscr{L}_{r, 0 ; 1}^{\Lambda, \Lambda_{0}}\right\|_{(a, b)}=0$, for $z \geqq 1$, and $\left\|\mathscr{L}_{r, 0 ; 1}^{\Lambda, \Lambda_{0}}\right\|_{(a, b)}=\left|\mathscr{L}_{r, 0 ; 1}^{\Lambda, \Lambda_{0}}\right|$, and 
similarly for $\mathscr{L}_{r, 1 ; 0}^{\Lambda, \Lambda_{0}}$. On the other hand, for $\Lambda \in\left[\Lambda_{1}, \Lambda_{0}\right]$ and $z \geqq 0$ we employ (2.26) and the bound $\left|\partial_{\Lambda} R(\Lambda, 0)\right| \leqq c \cdot \Lambda^{-3}$ to obtain, for $t=0,1$ and arbitrary $\eta \geqq 0$ :

$$
\begin{aligned}
& \left\|\partial_{\Lambda} \partial^{z} \mathscr{L}_{r, t ; n}^{\Lambda, \Lambda_{0}}\right\|_{(2 \Lambda, \eta)} \leqq \mathrm{const} \cdot\left\{\Lambda \cdot\left\|\partial^{z} \mathscr{L}_{r, t ; n+2}^{\Lambda, \Lambda_{0}}\right\|_{(2 \Lambda, \eta)}\right. \\
& +\sum_{r^{\prime}+r^{\prime \prime}=r} \Lambda^{-3} \cdot\left\|\mathscr{L}_{r^{\prime}, 0 ; 1}^{\Lambda, \Lambda_{0}}\right\|_{(2 \Lambda, \eta)} \cdot\left\|\partial^{z} \mathscr{L}_{r^{\prime \prime}, t ; n+1}^{\Lambda, \Lambda_{0}}\right\|_{(2 \Lambda, \eta)} \\
& +\sum_{\substack{r^{\prime}+r^{\prime \prime}=r \\
n^{\prime}+n^{\prime \prime}=n \\
z^{\prime}+z^{\prime \prime}+z^{\prime \prime \prime}=z}}^{*} \Lambda^{-3-z^{\prime \prime \prime}} \cdot\left\|\partial^{z^{\prime}} \mathscr{L}_{r^{\prime}, 0 ; n^{\prime}}^{\Lambda, \Lambda_{0}}\right\|_{(2 \Lambda, \eta)} \\
& \left.\cdot \| \partial^{z^{\prime \prime}} \mathscr{L}_{r^{\prime \prime}, t ; n^{\prime \prime}}^{\Lambda, \Lambda_{(2 \Lambda, \eta)}}\right\} \text {. } \\
& \left(\Lambda_{1} \leqq \Lambda \leqq \Lambda_{0}, \quad z \geqq 0\right)
\end{aligned}
$$

2.6. In this and the next subsection we will investigate in some detail the boundary conditions (bc) which the flow of effective Lagrangians obeys, i.e. we will take a closer look at $\mathscr{L}_{r, t ; n}^{\Lambda=\Lambda_{0}, \Lambda_{0}}$ and $\mathscr{L}_{r, t ; n}^{\Lambda=0, \Lambda_{0}}$. The ensuing results together with (2.27), (2.28) will turn out to be the main tools to prove perturbative renormalizability. As a start let us concentrate on the $t=0$ sector (the $t=1$ sector will be treated in the following subsection).

The general structure of the Green functions, $\mathscr{L}_{r, 0 ; n}^{\Lambda, \Lambda_{0}}$, of the $\Phi_{4}^{4}$ theory (without insertions of $B_{D}$ ) has been fixed by the Euclidean invariant bare interaction vertices, $l^{\Lambda_{0}}$, and the Euclidean free propagator, $C_{\Lambda}^{\Lambda_{0}}$. In particular $L_{r, 0}^{\Lambda_{0}, \Lambda_{0}}=l_{r}^{\Lambda_{0}}$, thus by (2.6)

$\underline{\Lambda=\Lambda_{0}}$ :

$$
\partial_{p}^{w} \mathscr{L}_{r, 0 ; n}^{\Lambda_{0}, \Lambda_{0}} \equiv 0, \quad \text { if } n+|w| \geqq 5 .
$$

From (2.18) and (2.20) we know that among the $\partial_{p}^{w} \mathscr{L}_{r, 0 ; n}^{\Lambda=0, \Lambda_{0}}(0), n+|w| \leqq 4$, at most the terms with $(n,|w|)=(1,0),(2,0),(2,2),(3,0),(4,0)$ are nonzero; furthermore $(2.21)$ exhibits the tensorial structure of the $(2,2)$ term. By comparison with (2.6) we conclude that we can always inductively adjust the bare parameters $a_{r}^{(j)}$, $1 \leqq j \leqq 5$, such that the not a priori vanishing relevant and marginal $\partial_{p}^{w} \mathscr{L}_{r, 0 ; n}^{0, \Lambda_{r}}(0)$ obey the following general renormalization conditions (i.e. bc at $\Lambda=0$ ), for $|r| \geqq 1$ :

$$
\begin{aligned}
& \underline{\Lambda=0}: \quad \text { a) } \mathscr{L}_{r, 0 ; 1}^{0, \Lambda_{0}}=a_{r}^{(1) R}, \\
& \text { b) } \mathscr{L}_{r, 0 ; 2}^{0, \Lambda_{0}}(0)=a_{r}^{(2) R}, \quad \partial_{p_{\mu}} \partial_{p_{v}} \mathscr{L}_{r, 0 ; 2}^{0, \Lambda_{0}}(0)=-\delta_{\mu, v} \cdot 2 \cdot a_{r}^{(3) R} \text {, } \\
& \text { c) } \mathscr{L}_{r, 0 ; 3}^{0, \Lambda_{0}}(0)=a_{r}^{(4) R} \text {, } \\
& \text { d) } \mathscr{L}_{r, 0 ; 4}^{0, \Lambda_{0}}(0)=a_{r}^{(5) R} \text {. }
\end{aligned}
$$

Here the $a_{r}^{(j) R}, 1 \leqq j \leqq 5,|r| \geqq 1$, are taken to be finite and (w.l.g.) $\Lambda_{0}$-independent, but otherwise they are arbitrary. It is not difficult to see, by induction in $|r|$, that once we require $(2.30)$ to hold the bare parameters $a_{r}^{(j)} \equiv a_{r}^{(j)}\left(a_{r^{\prime}}^{(i) R}, \Lambda_{0}, \ldots\right)$ are finite and well-defined, for $\Lambda_{0}<\infty$. Let us point out, finally, that the renormalization conditions (2.30) have been chosen at zero momentum for reasons of simplicity only; indeed arbitrary nonzero external momenta could have been taken as well [16]. 
For example, the standard BPHZ-type renormalization conditions to define a $\mathbb{Z}_{2}$-nonsymmetric $\Phi_{4}^{4}$ theory where $g_{1}, g_{2}$ can be interpreted as the "coupling constants" of the $\phi^{3}$ and $\phi^{4}$ vertices, respectively, amount to set $a_{r}^{(1) R}=a_{r}^{(2) R}=a_{r}^{(3) R}=0, a_{r}^{(4) R}=\frac{1}{3 !} \delta_{r,(1,0)}, a_{r}^{(5) R}=\frac{1}{4 !} \delta_{r,(0,1)}$. Obviously, renormalization conditions which define a $\mathbb{Z}_{2}$-symmetric theory could be imposed as well.

Summarizing we have shown that the bc (2.30) serve the following two purposes. First, for $\Lambda_{0}<\infty$ they lead to uniquely and well-defined bare parameters and thus Green functions of the $\Phi_{4}^{4}$ theory, and furthermore they guarantee that for $|r| \geqq 1$

$$
\partial_{p}^{w} \mathscr{L}_{r, 0 ; n}^{\Lambda=0, \Lambda_{0}}(0)=\text { finite and } \Lambda_{0} \text {-independent }, \quad \text { if } n+|w| \leqq 4
$$

2.7. We turn our attention now to the $t=1$ sector. Similarly as before the general structure of the $\Phi_{4}^{4}$ Green functions with one insertion of $B_{D}(x=0), \mathscr{L}_{r, 1 ; n}^{\Lambda, \Lambda_{0}}$, is prescribed by the vertices $(2.5),(2.6)$ and $(2.7)$ and by the covariance $C_{\Lambda}^{\Lambda_{0}}$. Once the renormalization conditions (2.30) are imposed the $a_{r}^{(j)}$ are known, but as yet the bare parameters $b_{r ; 0}, b_{r ; n,\{w\}}$ are undetermined. Nevertheless we infer from (2.7) and (2.22) that

$\underline{\Lambda=\Lambda_{0}}: \quad \partial_{p}^{w} \mathscr{L}_{r, 1 ; n}^{\Lambda_{0}, \Lambda_{0}} \equiv 0, \quad$ if $n+|w| \geqq D+1$.

In order to motivate the structure of the bc at $\Lambda=0$, i.e. of the renormalization conditions on the Green functions with one insertion of $B_{D}(x=0)$, we have to digress a bit. We begin by noting that the permutation symmetry

$$
\mathscr{L}_{r, 1 ; n}^{\Lambda, \Lambda_{0}}\left(p_{1}, \ldots, p_{n}\right)=\mathscr{L}_{r, 1 ; n}^{\Lambda, \Lambda_{0}}\left(p_{\pi(1)}, \ldots, p_{\pi(n)}\right), \quad \forall \pi \in S_{n},
$$

implies that for all $\Lambda$

$$
\partial_{p}^{w} \mathscr{L}_{r, 1 ; n}^{\Lambda, \Lambda_{0}}(0)=\partial_{p}^{w^{\prime}} \mathscr{L}_{r, 1 ; n}^{\Lambda, \Lambda_{0}}(0), \quad \text { if }\{w\}=\left\{w^{\prime}\right\},
$$

where we have used $w=\left(w_{1}, \ldots, w_{n}\right) \Rightarrow\{w\}:=\left\{w_{1}, \ldots, w_{n}\right\}$. Formulae (2.5)-(2.8) and (2.22) show that $\mathscr{L}_{r, 1 ; 0}^{\Lambda_{0}, \Lambda_{0}}=-b_{r ; 0}$ and that for $n \geqq 1$

$$
\mathscr{L}_{r, 1 ; n}^{\Lambda_{0}, \Lambda_{0}}=-\sum_{\{w\}: n+|w| \leqq D} b_{r ; n,\{w\}} \cdot\left[\left(i p_{1}\right)^{w_{1}} \ldots\left(i p_{n}\right)^{w_{n}}\right]_{\text {symm }} .
$$

Obviously

$$
\left.\partial_{p}^{w^{\prime}}\left[\left(i p_{1}\right)^{w_{1}} \ldots\left(i p_{n}\right)^{w_{n}}\right]_{\text {symm }}\right|_{p_{1}=\cdots=p_{n}=0} \sim \delta_{\{w\},\left\{w^{\prime}\right\}},
$$

so we may define the nonzero number $\mathscr{N}_{\{w\}}$ by

$$
\left.\partial_{p}^{w^{\prime}}\left[\left(i p_{1}\right)^{w_{1}} \ldots\left(i p_{n}\right)^{w_{n}}\right]_{\text {symm }}\right|_{p_{1}=\cdots=p_{n}=0}=\delta_{\{w\},\left\{w^{\prime}\right\}} \cdot \mathscr{N}_{\{w\}} \cdot
$$

With this notation we find for $n \geqq 1$

$$
\partial_{p}^{w} \mathscr{L}_{r, 1 ; n}^{\Lambda_{0}, \Lambda_{0}}(0)=-b_{r ; n,\{w\}} \cdot \mathcal{N}_{\{w\}}, \quad \text { if } n+|w| \leqq D
$$

And now (2.33), (2.36) tell us that it is consistent to impose the general renormalization conditions, for $|r| \geqq 0$,

$\Lambda=0$ :

a) $\mathscr{L}_{r, 1 ; 0}^{0, \Lambda_{0}}=-b_{r ; 0}^{R}$

b) $\partial_{p}^{w} \mathscr{L}_{r, 1 ; n}^{0, \Lambda_{0}}(0)=-b_{r ; n,\{w\}}^{R} \cdot \mathscr{N}_{\{w\}}, \quad$ if $n+|w| \leqq D \quad$ and $\quad n_{1} \geqq 1$. 
As in the sector $t=0$ the renormalization constants $b_{r ; 0}^{R}$ and $b_{r ; n,\{w\}}^{R}$ are supposed to be finite and $\Lambda_{0}$-independent. It is not difficult to check that together with the $a_{r}^{(j) R}$ they determine uniquely the bare parameters $b_{r ; 0}$ and $b_{r ; n,\{w\}}$ and that these are finite, for $\Lambda_{0}<\infty$. Again zero external momentum (to fix the renormalization condition) has been chosen for convenience only. Notice, finally, that we do not require the renormalization condition (2.37) to exhibit a Euclidean invariant structure.

The most common renormalization conditions (2.37) are probably those which lead to Zimmermann's normal products $[1,2]$; we postpone their discussion to Sect. 4.

\section{Perturbative Renormalizability}

In Sect. 2 we have collected nearly enough information to prove perturbative renormalizability; the missing piece is the definition

$$
\theta_{\min }:=1+\max \{3, D\},
$$

which ensures that $\partial_{p}^{w} \mathscr{L}_{r, t ; n}^{\Lambda, \Lambda_{0}} \in C\left(\left[0, \Lambda_{0}\right] \times \mathbb{R}^{4(n+t-1)}\right)$, for all $w$ with $0 \leqq|w| \leqq$ $\max \{3, D\}$; in fact continuity holds at least for all $w$ with $0 \leqq|w| \leqq \theta-1$. $^{2}$

Choose an arbitrary $\eta, 0 \leqq \eta<\infty$, but keep $\eta$ fixed in what follows. $\eta$ is meant to indicate the range of the momenta $p_{1}, p_{2}, \ldots$ within which the convergence, as $\Lambda_{0} \rightarrow \infty$, of $\mathscr{L}_{r, t ; n}^{0, \Lambda_{0}}\left(p_{1}, p_{2}, \ldots\right)$ will be proved.

Theorem 3 (Boundedness). For the general renormalization conditions (2.30) and (2.37), for any fixed $\eta, 0 \leqq \eta<\infty$, and for all $z$ with $0 \leqq z \leqq \theta-1$ we find that the $\Phi_{4}^{4}$ Green functions obey the bounds

$$
\left\|\partial^{z} \mathscr{L}_{r, 0 ; n}^{\Lambda, \Lambda_{0}}\right\|_{(2 \Lambda, \eta)} \leqq \begin{cases}\text { const }, & 0 \leqq \Lambda \leqq \Lambda_{1} \\ \Lambda^{4-n-z} \cdot P \log \left(\frac{\Lambda}{\Lambda_{1}}\right), & \Lambda_{1} \leqq \Lambda \leqq \Lambda_{0}\end{cases}
$$

and that the $\Phi_{4}^{4}$ Green functions with one insertion of $B_{D}(0)$ are bounded as

$$
\left\|\partial^{z} \mathscr{L}_{r, 1 ; n}^{\Lambda, \Lambda_{0}}\right\|_{(2 \Lambda, \eta)} \leqq \begin{cases}\text { const }, & 0 \leqq \Lambda \leqq \Lambda_{1} \\ \Lambda^{D-n-z} \cdot P \log \left(\frac{\Lambda}{\Lambda_{1}}\right), & \Lambda_{1} \leqq \Lambda \leqq \Lambda_{0}\end{cases}
$$

Here "const" stands for some finite number which depends neither on $\Lambda$ nor on $\Lambda_{0}$, but which in general will depend on $\eta, \Lambda_{1}, r, t, n, z, \ldots ; P \log \left(\Lambda / \Lambda_{1}\right)$ is some polynomial in $\log \left(\Lambda / \Lambda_{1}\right)$ with coefficients which again depend neither on $\Lambda$ nor on $\Lambda_{0}$ but which may depend on $\eta, \Lambda_{1}, r, t, \ldots$

Proof. (I) $t=0$. The proof of (3.2) can, in principle, be found in ref. [16]; for the sake of completeness, however, we will reproduce it here. Equation (3.2) will be proved by induction, the induction scheme being the one which has been invented in ref. [15].

${ }^{2}$ For the purpose of analyzing the $t=0$ sector only, the definition $\theta_{\min }:=4$ would have been sufficient. 
The induction hypothesis is that (3.2) holds for $\{(r, n, z):((|r|<a) \wedge(n \in \mathbb{N})) \vee$ $((|r|=a) \wedge(n>b)), 0 \leqq z \leqq \theta-1\}$. The induction step consists in proving (3.2) for $\{(r, n, z):(|r|=a) \wedge(n=b), 0 \leqq z \leqq \theta-1\}$.

Obviously the induction hypothesis is fulfilled for $a=1, b=4$ (because $\mathscr{L}_{(0,0), 0 ; n}^{\Lambda, \Lambda_{0}} \equiv 0$ and because of $\left.(2.19)\right)$, so we may start the induction procedure at $(a, b)=(1,4)$. Moreover, once we have arrived at and dealt with $(a, b)=(a, 1)$ the induction hypothesis becomes automatically obeyed by $\left(a^{\prime}, b^{\prime}\right):=$ $(a+1,2(a+1)+2)$, again because of (2.19). We conclude that with this induction scheme we reach all nontrivial $(a, b)$.

Let us carry out the induction step for the pair $(a, b)$; i.e. we wish to check (3.2) for $\{(r, n, z):((|r|=a) \wedge(n=b)), 0 \leqq z \leqq \theta-1\}$.

Choose and fix $r$ with $|r|=a$. We first show (3.2) for those $z$ with $b+z \geqq 5$. If $b=1$ then $z \geqq 4$; but since $\partial_{p} \mathscr{L}_{r, 0 ; 1}^{\Lambda, \Lambda_{0}} \equiv 0$ (3.2) obviously holds. So assume that $b \geqq 2$; and note that for $b \geqq 2\{z: 0 \leqq z \leqq \theta-1\} \cap\{z: b+z \geqq 5\} \supset\{3\}$, because of (3.1). Use (2.29) to write

$$
\begin{aligned}
\left\|\partial^{z} \mathscr{L}_{r, 0 ; b}^{\Lambda, \Lambda_{0}}\right\|_{(2 \Lambda, \eta)} & =\left\|\partial^{z}\left(\mathscr{L}_{r, 0 ; b}^{\Lambda, \Lambda_{0}}-\mathscr{L}_{r, 0 ; b}^{\Lambda_{0}, \Lambda_{0}}\right)\right\|_{(2 \Lambda, \eta)} \\
& \leqq \int_{\Lambda}^{\Lambda_{0}} d \Lambda^{\prime}\left\|\partial_{\Lambda^{\prime}} \partial^{z} \mathscr{L}_{r, 0 ; b}^{\Lambda^{\prime}, \Lambda_{0}}\right\|_{\left(2 \Lambda^{\prime}, \eta\right)} .
\end{aligned}
$$

If $\Lambda \in\left[\Lambda_{1}, \Lambda_{0}\right]$ the r.h.s. of (3.4) is now estimated by replacing the integrand by the corresponding r.h.s. of (2.28); now employ the induction hypothesis on (2.28) to arrive at $^{3}$

$$
\begin{aligned}
\left\|\partial^{z} \mathscr{L}_{r, 0 ; b}^{\Lambda, \Lambda_{0}}\right\|_{(2 \Lambda, \eta)} & \leqq \int_{\Lambda}^{\Lambda_{0}} d \Lambda^{\prime} \Lambda^{\prime 3-b-z} \cdot P \log \left(\frac{\Lambda^{\prime}}{\Lambda_{1}}\right) \\
& \leqq \Lambda^{4-b-z} \cdot\left(P \log \left(\frac{\Lambda}{\Lambda_{1}}\right)+\left(\frac{\Lambda}{\Lambda_{0}}\right) \cdot P \log \left(\frac{\Lambda_{0}}{\Lambda_{1}}\right)\right) \\
& \leqq \Lambda^{4-b-z} \cdot P \log \left(\frac{\Lambda}{\Lambda_{1}}\right)
\end{aligned}
$$

On the other hand, if $\Lambda \in\left[0, \Lambda_{1}\right]$ we split, in (3.4), $\int_{\Lambda}^{\Lambda_{0}}=\int_{\Lambda}^{\Lambda_{1}}+\int_{\Lambda_{1}}^{\Lambda_{0}}$; the second integral has already been estimated in (3.5), and the first is easily estimated by using (2.27) and the induction hypothesis; this gives (3.2).

Now we are going to verify (3.2) for those $z$ with $b+z \leqq 4$. The method is induction in $z$. Induction hypothesis: (3.2) holds for all $z$ with $z^{\prime}<z \leqq \theta-1$. This hypothesis is certainly true for $z^{\prime}=0$, if $b=1$, and for $z^{\prime}$ with $b+z^{\prime}=4$, if $b \geqq 2$ (as we have just shown before). Let us prove now (3.2) for $z=z^{\prime}$ thereby completing one induction step.

Obviously, for any $w$ with $|w|=z^{\prime}$ we have

$$
\left|\partial_{p}^{w} \mathscr{L}_{r, 0 ; b}^{\Lambda, \Lambda_{0}}(0)-\partial_{p}^{w} \mathscr{L}_{r, 0 ; b}^{0, \Lambda_{0}}(0)\right| \leqq \int_{0}^{\Lambda} d \Lambda^{\prime}\left\|\partial_{\Lambda^{\prime}} \partial^{z^{\prime}} \mathscr{L}_{r, 0 ; b}^{\Lambda^{\prime}, \Lambda_{0}}\right\|_{\left(2 \Lambda^{\prime}, \eta\right)}
$$

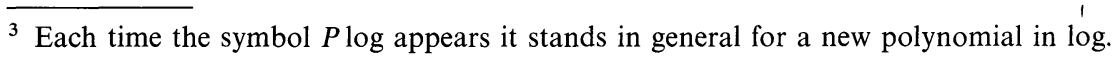


and therefore, using (2.31),

$$
\left|\partial_{p}^{w} \mathscr{L}_{r, 0 ; b}^{\Lambda, \Lambda_{0}}(0)\right| \leqq \text { const }+\int_{0}^{\Lambda} d \Lambda^{\prime}\left\|\partial_{\Lambda^{\prime}} \partial^{z^{\prime}} \mathscr{L}_{r, 0 ; b^{\prime}}^{\Lambda^{\prime}, \Lambda_{0}}\right\|_{\left(2 \Lambda^{\prime}, \eta\right)} .
$$

The r.h.s. of (3.6) is estimated by replacing again the integrand by the r.h.s. of (2.27) or (2.28) which in turn are estimated using the induction hypothesis (of the $(a, b)$ induction procedure). We thus obtain

$$
\mid \partial_{p}^{w} \mathscr{L}_{r, 0 ; b}^{\Lambda, \Lambda_{0}(0) \mid \leqq} \begin{cases}\text { const }, & 0 \leqq \Lambda \leqq \Lambda_{1} \\ \Lambda^{4-b-z^{\prime}} \cdot P \log \left(\frac{\Lambda}{\Lambda_{1}}\right), & \Lambda_{1} \leqq \Lambda \leqq \Lambda_{0}\end{cases}
$$

For $b=1$ (3.7) implies (3.2); and for $b \geqq 2$ Taylor's formula

$$
\begin{aligned}
\partial_{p}^{w} \mathscr{L}_{r, 0 ; b}^{\Lambda, \Lambda_{0}}\left(p_{1}, \ldots, p_{b-1}\right)= & \partial_{p}^{w} \mathscr{L}_{r, 0 ; b}^{\Lambda, \Lambda_{0}(0)} \\
& +\sum_{i=1}^{b-1} \sum_{\mu=1}^{4} p_{i, \mu} \int_{0}^{1} d \lambda \partial_{k_{k, \mu}} \partial_{k}^{w} \mathscr{L}_{r, 0 ; b}^{\Lambda, \Lambda_{0}}\left(k_{1}, \ldots, k_{b-1}\right),
\end{aligned}
$$

where $k_{j}:=\lambda \cdot p_{j}, 1 \leqq j \leqq b-1$, together with (3.7) (to bound the first term on the r.h.s. of (3.8)) and with the induction hypothesis in $z$ (to estimate the integrand in (3.8)) finally gives (3.2) for $\left(r, b, z^{\prime}\right)$.

(II) $t=1$. The inequalities (3.3) will be verified by induction. Because all the details of the inductive proof are very similar to those encountered in the $t=0$ sector we will be quite brief.

Induction hypothesis: (3.3) holds for $\left\{(r, n, z):\left((|r|<a) \wedge\left(n \in \mathbb{N}_{0}\right)\right) \vee((|r|=a)\right.$ $\wedge(n>b)), 0 \leqq z \leqq \theta-1\}$. Induction step: Prove (3.3) for $\{(r, n, z):(|r|=a) \wedge$ $(n=b), 0 \leqq z \leqq \theta-1\}$. It is easy to check that $\mathscr{L}_{r, 1 ; n}^{\Lambda, \Lambda_{0}} \equiv 0$, if $n>2|r|+D$; thus the induction hypothesis is true for $(a, b)=(0, D)$, and once we have treated $(a, b)=(a, 0)$ the inductive process may be continued with $\left(a^{\prime}, b^{\prime}\right):=$ $(a+1,2(a+1)+D)$.

We now sketch the induction step at $(a, b)$. Consider first those $z$ with $b+z \geqq D+1$. The case $b=0, z \geqq D+1$ is trivial; so assume $b \geqq 1$. Note that because of (3.1) we have $\{z: 0 \leqq z \leqq \theta-1, b+z \geqq D+1\} \supset\{D\}$. Using the bc (2.32) we get in analogy to (3.4)

$$
\left\|\partial^{z} \mathscr{L}_{r, 1 ; b}^{\Lambda, \Lambda_{0}}\right\|_{(2 \Lambda, \eta)} \leqq \int_{\Lambda}^{\Lambda_{0}} d \Lambda^{\prime}\left\|\partial_{\Lambda^{\prime}} \partial^{z} \mathscr{L}_{r, i ; b}^{\Lambda^{\prime}, \Lambda_{0}}\right\|_{\left(2 \Lambda^{\prime}, \eta\right)} .
$$

(2.27), (2.28), (3.2) and the induction hypothesis now yield (3.3).

So let us turn to those $z$ with $b+z \leqq D$. Induction hypothesis: (3.3) has been checked for all $z$ with $z^{\prime}<z \leqq \theta-1$. Induction step: Prove (3.3) for $z=z^{\prime}$. The induction hypothesis clearly holds for $z^{\prime}=0$, if $b=0$, and for $z^{\prime}$ with $b+z^{\prime}=D$, if $b \geqq 1$. Now remember (2.37) to conclude that for all $w$ with $|w|=z^{\prime}$

$$
\left|\partial_{p}^{w} \mathscr{L}_{r, 1 ; b}^{\Lambda, \Lambda_{0}}(0)\right| \leqq \text { const }+\int_{0}^{\Lambda} d \Lambda^{\prime}\left\|\partial_{\Lambda^{\prime}} \partial^{z^{\prime}} \mathscr{L}_{r, 1 ; b^{\Lambda^{\prime}}, \Lambda_{0}}\right\|_{\left(2 \Lambda^{\prime}, \eta\right)} .
$$

Use (2.27), (2.28), (3.2), the (a,b)-induction hypothesis as well as Taylor's formula and the $z$-induction hypothesis to verify (3.3). 
Theorem 4 (Convergence). Under the same conditions as in Theorem 3 we have for $t=0,1$

$\left\|\partial_{\Lambda_{0}} \partial^{z} \mathscr{L}_{r, t ; n}^{\Lambda, \Lambda_{0}}\right\|_{(2 \Lambda, \eta)} \leqq \begin{cases}\Lambda_{0}^{-2} \cdot P \log \left(\frac{\Lambda_{0}}{\Lambda_{1}}\right), & 0 \leqq \Lambda \leqq \Lambda_{1} \\ \Lambda_{0}^{-2} \cdot \Lambda^{4(1-t)+D \cdot t-n-z+1} \cdot P \log \left(\frac{\Lambda_{0}}{\Lambda_{1}}\right), & \Lambda_{1} \leqq \Lambda \leqq \Lambda_{0} .\end{cases}$

Proof. We employ the induction procedure described in the proof of Theorem 3 .

If $b+z \geqq 4(1-t)+D \cdot t+1$ : Replace $n \mapsto b$ in (2.23); integrate (2.23) now from $\Lambda, 0 \leqq \Lambda<\Lambda_{0}$, up to $\Lambda_{0}$ and apply $\partial_{p}^{w},|w|=z$; use (2.29) or (2.32); upon acting with $\partial_{\Lambda_{0}}$ on the resulting equation one obtains

$$
\begin{aligned}
-\partial_{\Lambda_{0}} \partial_{p}^{w} \mathscr{L}_{r, t ; b}^{\Lambda, \Lambda_{0}}= & \left.\partial_{p}^{w} \text { (r.h.s. of }(2.23)\right)\left.\right|_{\Lambda=\Lambda_{0}} \\
& \left.+\int_{\Lambda}^{\Lambda_{0}} d \Lambda^{\prime} \partial_{\Lambda_{0}} \partial_{p}^{w} \text { (r.h.s. of }(2.23)\right)\left.\right|_{\Lambda=\Lambda^{\prime}} ;
\end{aligned}
$$

the first term on the r.h.s. of (3.12) can be estimated by the bounds (3.2), (3.3); the integrand in the second term is estimated using (3.2), (3.3) as well as the induction hypothesis.

If $b+z \leqq 4(1-t)+D \cdot t$ : Apply $\partial_{p}^{w}$, at $p_{1}=\cdots=p_{b+t-1}=0,|w|=z^{\prime}$, on (2.23) and integrate the result from 0 up to $\Lambda, 0 \leqq \Lambda<\Lambda_{0}$; remember that the bc (2.30), (2.37) are $\Lambda_{0}$-independent; now apply $\partial_{\Lambda_{0}}$ to obtain

$$
\left.\partial_{\Lambda_{0}} \partial_{p}^{w} \mathscr{L}_{r, t ; b}^{\Lambda, \Lambda_{0}}(0)=\int_{0}^{\Lambda} d \Lambda^{\prime} \partial_{\Lambda_{0}} \partial_{p}^{w} \text { (r.h.s. of }(2.23)\right)\left.\right|_{\Lambda^{\prime} ; p_{1}=\cdots=0} ;
$$

as before the r.h.s. of (3.13) is bounded with the help of (3.2), (3.3) and of the induction hypothesis; use Taylor's formula and the induction in $z$ to arrive at (3.11).

Corollary. Under the conditions specified in Theorem 3 perturbative renormalizability holds for $t=0$, 1 ; i.e. for $\left|p_{i}\right| \leqq \eta, 1 \leqq i \leqq n+t-1$, the limit

$$
\lim _{\Lambda_{0} \rightarrow \infty} \mathscr{L}_{r, t ; n}^{0, \Lambda_{0}}\left(p_{1}, \ldots, p_{n+t-1}\right)
$$

exists and is $C^{(\theta-1)}$ (in the momenta $p_{1}, \ldots, p_{n+t-1}$ ).

One major ingredient in the proofs of Theorem 3 and 4 has been the estimated form of the differential flow equation (2.23), estimated for all $\Lambda \in\left[0, \Lambda_{0}\right]$. The unpleasant regulator $\theta$ has been necessary in order to obtain useful bounds also as $\Lambda \rightarrow 0 .{ }^{4}$ Of course one expects that $\theta$ carries no physical significance, but a proof of this would be welcome. Due to the well known technical reasons we cannot admit, with our method, that $\theta<\theta_{\min }$. So for the moment the best we can do is to compare the Green functions which are obtained with the help of $\theta$ and $\theta^{\prime}$, $\theta, \theta^{\prime} \geqq \theta_{\min }$, namely $\mathscr{L}_{r, t ; n}^{\Lambda, \Lambda_{o}}(\vec{p} ; \theta)$ and $\mathscr{L}_{r, t ; n}^{\Lambda, \Lambda_{o}}\left(\vec{p} ; \theta^{\prime}\right)$, where $\vec{p} \equiv\left(p_{1}, \ldots, p_{n+t-1}\right)$.

\footnotetext{
${ }^{4}$ In a somewhat different approach [16] it is sufficient to estimate (2.23) for $\Lambda \in\left[\Lambda_{1}, \Lambda_{0}\right]$; this technical advantage is, unfortunately, traded for inconveniences in connection with the evaluation of the information contained in the bc at $\Lambda=0$. We believe that the method in the present paper is aesthetically more appealing.
} 
Theorem 5. For $\theta \geqq \theta_{\min }$ and $t=0,1$ we have

$$
\left\|\partial_{\theta} \mathscr{L}_{r, t ; n}^{0, \Lambda_{0}}\right\|_{(0, \eta)} \leqq \text { const } \cdot e^{-\Lambda_{0} / m} .
$$

The proof is given in the Appendix. (3.15) confirms our expectations and shows, moreover, that the renormalized Green functions (3.14) are $C^{\infty}$.

\section{Zimmermann Identities, Lowenstein Rule}

4.1. Let $\left\{f_{r, 1 ; n}^{\Lambda, \Lambda_{0}}\left(p_{1}, \ldots, p_{n}\right):|r| \geqq 0, n \geqq 0, \Lambda \in\left[0, \Lambda_{0}\right], p_{i} \in \mathbb{R}^{4}, 1 \leqq i \leqq n\right\}$ be a set of functions which obey the differential flow equation (2.23), i.e. the $f_{r, 1 ; n}^{A, \bar{\Lambda}_{0}}$ satisfy the linear differential equation

$$
\begin{aligned}
& \partial_{\Lambda} f_{r, 1 ; n}^{\Lambda, \Lambda_{0}}\left(p_{1}, \ldots, p_{n}\right)=-\left(\begin{array}{c}
n+2 \\
2
\end{array}\right) \int \frac{d^{4} q}{(2 \pi)^{4}} \frac{\partial_{\Lambda} R(\Lambda, q)}{q^{2}+m^{2}} \\
& \cdot f_{r, 1 ; n+2}^{\Lambda, \Lambda_{0}}\left(q,-q, p_{1}, \ldots, p_{n}\right) \\
& +\sum_{r^{\prime}+r^{\prime \prime}=r}(n+1) \cdot \frac{\partial_{\Lambda} R(\Lambda, 0)}{m^{2}} \cdot \mathscr{L}_{r^{\prime}, 0 ; 1}^{\Lambda, \Lambda_{0}} \\
& \cdot f_{r^{\prime \prime}, 1 ; n+1}^{\Lambda, \Lambda_{0}}\left(0, p_{1}, \ldots, p_{n}\right) \\
& +\sum_{\substack{r^{\prime}+r^{\prime \prime}=r \\
n^{\prime}+n^{\prime \prime}=n+2 \\
n^{\prime} \geqq 2, n^{\prime \prime} \geqq 1}} n^{\prime} n^{\prime \prime}\left[\frac{\partial_{\Lambda} R(\Lambda, Q)}{Q^{2}+m^{2}} \cdot \mathscr{L}_{r^{\prime}, 0 ; n^{\prime}}^{\Lambda, \Lambda_{0}}\left(p_{1}, \ldots, p_{n^{\prime}-1}\right)\right. \\
& \left.\cdot f_{r^{\prime \prime}, 1 ; n^{\prime \prime}}^{\Lambda, \Lambda_{0}}\left(-Q, p_{n^{\prime}}, \ldots, p_{n}\right)\right]_{\mathrm{symm}} .
\end{aligned}
$$

Here the $\mathscr{L}_{r, 0 ; n}^{\Lambda, \Lambda_{0}}$ are the familiar $\Phi_{4}^{4}$ Green functions, regularized with the help of some $\theta \geqq 4$ (it is irrelevant which $\theta$, see Theorem 5) and obeying the renormalization conditions (2.30).

Lemma 6. $f_{r, 1 ; n}^{\Lambda, \Lambda_{0}} \equiv 0, \forall r, n$, iff the following four conditions are fulfilled:

a) $\exists d \geqq 1$ such that $f_{r, 1 ; n}^{\Lambda, \Lambda_{0}} \in C^{(d)}\left(\left[0, \Lambda_{0}\right] \times \mathbb{R}^{4 n}\right), \forall r, n$;

b) $\partial_{p}^{w} \hat{f}_{r, 1 ; n}^{\Lambda_{0}, \Lambda_{0}} \equiv 0, \forall r$, if $(0 \leqq|w| \leqq d) \wedge(n+|w| \geqq d+1)$;

c) $\partial_{p}^{w} f_{r, 1 ; n}^{0, \Lambda_{0}}(0)=0, \forall r$, if $n+|w| \leqq d$;

d) for each $z \geqq 0$ there exists $n(z), 0 \leqq n(z)<\infty$, such that $f_{r, 1 ; n}^{\Lambda, \Lambda_{0}} \equiv 0$ if $(|r|=z) \wedge$ $(n>n(z))$.

Proof. " $\Rightarrow "$ : trivial. " $\Leftarrow$ ": Due to d) it is possible to apply the usual induction scheme. The induction hypothesis implies that for $|r|=a, n=b$ the r.h.s. of (4.1) vanishes. So $f_{r, 1 ; b}, \Lambda_{0}$ is independent of $\Lambda$. Use b) and c) and Taylor's formula to show that therefore $f_{r, 1 ; b}^{A, \Lambda_{0}} \equiv 0$.

This simple lemma will play one of the key roles in the derivation of the Zimmermann identities.

Let $\left\{B^{(j)}: 0 \leqq j \leqq N\right\}$ be a finite set of polynomials of the type (2.7), $B^{(j)}$ having dimension $D^{(j)} \geqq 1$. Put $\theta_{\min , N}:=1+\max \left\{3, D^{(0)}, \ldots, D^{(N)}\right\}$. Obviously $\theta_{\text {min }, N} \geqq \theta_{\text {min }}^{(j)} \equiv 1+\max \left\{3, D^{(j)}\right\}, \forall j$. Thus it is legitimate to choose some $\theta, \theta \geqq \theta_{\min , N}$, and to define $\mathscr{L}_{r, 0 ; n}^{1, \Lambda_{0}}$ to be those $\Phi_{4}^{4}$ Green functions which are 
regularized with $\theta$ and which obey arbitrary but fixed renormalization conditions (2.30) thereby fixing uniquely the bare $\Phi_{4}^{4}$ interaction $l^{\Lambda_{0}},(2.6)$. Furthermore, for $0 \leqq j \leqq N$ the $\mathscr{L}_{r, 1 ; n}^{\Lambda, \Lambda_{0}(j)}$ are defined as the Green functions which are regularized with the same $\theta$ and which contain one insertion of $B^{(j)}(x=0)$ into our fixed $\Phi_{4}^{4}$ theory; for each $j$ the $\mathscr{L}_{r, 1 ; n}^{\Lambda, \Lambda_{0}(j)}$ are supposed to obey some renormalization conditions (2.37). So in particular $\lim _{\Lambda_{0} \rightarrow \infty} \mathscr{L}_{r, 1 ; n}^{0, \Lambda_{0}(j)}$ exists, $\forall j$.

Let $c^{(j)}, 0 \leqq j \leqq N$, be fps in $g$ with coefficients $c_{r}^{(j)}$ which do not depend on $\Lambda, n, p_{1}, \ldots, p_{n}$, but which may depend on $\Lambda_{0}, R, \ldots$ We define $\mathscr{F}_{r, 1 ; n}$ by

$$
\mathscr{F}_{r, 1 ; n}^{\Lambda, \Lambda_{0}}\left(p_{1}, \ldots, p_{n}\right):=\sum_{\substack{r^{\prime}+r^{\prime \prime}=r \\ 0 \leqq j \leqq N}} c_{r^{\prime}}^{(j)} \cdot \mathscr{L}_{r^{\prime \prime}, 1 ; n}^{\Lambda, \Lambda_{0}(j)}\left(p_{1}, \ldots, p_{n}\right) .
$$

It is evident that $\mathscr{F}_{r, 1 ; n}^{\Lambda, \Lambda_{0}} \in C^{(\theta-1)}\left(\left[0, \Lambda_{0}\right] \times \mathbb{R}^{4 n}\right)$. Now apply $\partial_{\Lambda}$ on $\mathscr{F}_{r, 1 ; n}^{\Lambda, \Lambda_{0}}$, use the above assumptions on $c_{r}^{(j)}$ and employ (2.23) with $t=1$. In this way one easily checks the

Lemma 7. $\left\{\mathscr{F}_{r, 1 ; n}^{\Lambda, \Lambda_{0}}\right\}$ satisfies (4.1).

Note that $\mathscr{F}_{r, 1 ; n}^{\Lambda, \Lambda_{0}}$ fulfills condition d) of Lemma 6 (because each $\mathscr{L}_{r, 1 ; n}^{\Lambda, \Lambda_{0}(j)}$ does so); therefore, if we manage to adjust the coefficients $c_{r}^{(j)}$ such that also the conditions a)-c) are obeyed, the linear combination $\mathscr{F}_{r, 1 ; n}^{\Lambda, \Lambda_{0}}$ is identically zero. It might be interesting to investigate the ensuing conditions on $B^{(j)}$ and $c^{(j)}$ in full generality; however, for the purpose of establishing the generalized Zimmermann identities it is sufficient to consider, in what follows, only the special case $D^{(0)} \leqq D^{(1)}=D^{(2)}=\cdots=D^{(N)}$. We set

$$
D_{0}:=D^{(0)}, \quad D_{1}:=D^{(1)}=D^{(2)}=\cdots=D^{(N)},
$$

and assume that $N$ depends on $D_{1}$ in such a way that

$$
N=\left|\left\{(n,\{w\}): 1 \leqq n, n+|w| \leqq D_{1}\right\}\right| \text {. }
$$

We define the square matrix $\mathscr{M}$, with matrix elements $\mathscr{M}_{(n,\{w\})}^{(j)}$, by

$$
\mathscr{M}_{(n,\{w\})}^{(j)}:=\partial_{p}^{w} \mathscr{L}_{r=0,1 ; n}^{0, \Lambda_{0}(j)}(0), \quad 1 \leqq j \leqq N, \quad 1 \leqq n, \quad n+|w| \leqq D_{1} .
$$

Theorem 8 (Generalized Zimmermann identities). Assume that the renormalization conditions (2.37) on $\mathscr{L}_{r, 1 ; n}^{\Lambda, \Lambda_{0}(j)}, 0 \leqq j \leqq N$, have been chosen such that

a) $\mathscr{L}_{r, 1 ; 0}^{0, \Lambda_{0}(j)}=0, \quad 0 \leqq j \leqq N, \quad|r| \geqq 0$;

b) $\mathscr{M}=$ regular .

Then there exist uniquely determined (complex) numbers $c_{r}^{(j)}, 1 \leqq j \leqq N$, so that

$$
\mathscr{L}_{r, 1 ; n}^{\Lambda, \Lambda_{0}(0)}=\sum_{\substack{r^{\prime}+r^{\prime \prime}=r \\ 1 \leqq j \leqq N}} c_{r^{\prime}}^{(j)} \cdot \mathscr{L}_{r^{\prime \prime}, 1 ; n}^{\Lambda, \Lambda_{0}(j)}
$$

The $c_{r}^{(j)}$ have finite limits as $\Lambda_{0} \rightarrow \infty$.

Proof. We have to verify that a)-c) of Lemma 6 hold for

$$
\mathscr{F}_{r, 1 ; n}^{\Lambda, \Lambda_{0}}:=\mathscr{L}_{r, 1 ; n}^{\Lambda, \Lambda_{0}(0)}-\sum_{\substack{r^{\prime}+r^{\prime \prime}=r \\ 1 \leqq j \leqq N}} c_{r^{\prime}}^{(j)} \cdot \mathscr{L}_{r^{\prime \prime}, 1 ; n}^{\Lambda, \Lambda_{0}(j)}
$$

if the $c_{r}^{(j)}$ are chosen appropriately. 
Putting $d:=D_{1}$ we see that part a), and because of (2.32) and $D_{0} \leqq d$ also part b), of Lemma 6 hold true, for any choice of $c_{r}^{(j)}$. So we are left with checking condition $\mathrm{c}$ ).

We proceed by induction in $|r|$. Induction hypothesis: There is a unique choice of $\left\{c_{r}^{(j)}: 0 \leqq|r|<z, 1 \leqq j \leqq N\right\}$ such that $\left.\mathrm{c}\right)$ is fulfilled for all $(r, n, w)$ with $|r|<z$; moreover $\lim _{\Lambda_{0} \rightarrow \infty} c_{r}^{(j)}$ exists, for $0 \leqq|r|<z$ and $1 \leqq j \leqq N$. Induction step: Choose an $r$ with $|r|=z$. Equation (4.6) guarantees that $\partial_{p}^{w} \mathscr{F}_{r, 1 ; n=0}^{0, \Lambda_{0}}=0$, for all $w$ and $c_{r}^{(j)}$, so let us consider $n \geqq 1$. Now, c) is valid (for $n \geqq 1$ ) iff for all $w$ with $n+|w| \leqq D_{1}$

$$
\begin{aligned}
\partial_{p}^{w} \mathscr{L}_{r, 1 ; n}^{0, \Lambda_{0}(0)}(0)= & \sum_{\substack{r^{\prime}+r^{\prime \prime}=r \\
1 \leqq j \leqq N}} c_{r^{\prime}}^{(j)} \cdot \partial_{p}^{w} \mathscr{L}_{r^{\prime \prime}, 1 ; n}^{0, \Lambda_{0}(j)}(0) \\
= & \sum_{\substack{r^{\prime}+r^{\prime \prime}=r \\
\left|r^{\prime \prime}\right|>0 \\
1 \leqq j \leqq N}} c_{r^{\prime}}^{(j)} \cdot \partial_{p}^{w} \mathscr{L}_{r^{\prime \prime}, 1 ; n}^{0, \Lambda_{0}(j)}(0)+\sum_{1 \leqq j \leqq N} c_{r}^{(j)} \cdot \mathscr{M}_{(n,\{w\})}^{(j)} .
\end{aligned}
$$

The induction hypothesis controls the first sum (in the second line) on the r.h.s. of (4.9), and (4.7) enables us to invert $\mathscr{M}$ and thus to complete the induction step.

4.2. As a first application of Theorem 8 we are going to derive our version of the standard Zimmermann identities [1,2], but in order to do that we first have to introduce our version of Zimmermann's normal products (cf. also the discussion in the introductory section).

For $D \geqq n^{\prime}+\left|w^{\prime}\right|$ let $B_{D,\left(n^{\prime},\left\{w^{\prime}\right\}\right)}$ be the unique polynomial such that the connected amputated Green functions with one insertion of $B_{D,\left(n^{\prime},\left\{w^{\prime}\right\}\right)}(x=0)$, $\mathscr{L}_{r, 1 ; n}^{\Lambda, \Lambda_{0}}\left(B_{D,\left(n^{\prime},\left\{w^{\prime}\right\}\right)}(0) ; p_{1}, \ldots, p_{n}\right)$, obey the renormalization conditions (2.37) with

$$
\begin{aligned}
b_{r ; 0}^{R} & :=0, \quad \forall r, \\
b_{r, n,\{w\}}^{R} & :=\delta_{n, n^{\prime}} \cdot \delta_{\{w\},\left\{w^{\prime}\right\}} \cdot \delta_{r,(0,0)} .
\end{aligned}
$$

We introduce the abbreviation $M_{n,\{w\}}(x):=\partial_{x}^{w_{1}} \phi(x) \ldots \partial_{x}^{w_{n}} \phi(x)$. Now our Zimmermann normal product of dimension (or subtraction degree) $D$ of the monomial $M_{n^{\prime},\left\{w^{\prime}\right\}}(x), N_{D}^{c}\left[M_{n^{\prime},\left\{w^{\prime}\right\}}(x)\right], D \geqq n^{\prime}+\left|w^{\prime}\right|$, is defined by requiring that the connected amputated Green functions with one insertion of $N_{D}^{c}\left[M_{n^{\prime},\left\{w^{\prime}\right\}}(x)\right]$, $\mathscr{L}_{r, 1 ; n}^{\Lambda, \Lambda_{0}}\left(N_{D}^{c}\left[M_{n^{\prime},\left\{w^{\prime}\right\}}(x)\right] ; p_{1}, \ldots, p_{n}\right)$, obey

$$
\mathscr{L}_{r, 1 ; n}^{\Lambda, \Lambda_{0}}\left(N_{D}^{c}\left[M_{n^{\prime},\left\{w^{\prime}\right\}}(x)\right] ; \ldots\right):=\mathscr{L}_{r, 1 ; n}^{\Lambda, \Lambda_{0}}\left(B_{D,\left(n^{\prime},\left\{w^{\prime}\right\}\right)}(x) ; \ldots\right) .
$$

The Zimmermann normal products $N_{D}^{c}\left[M_{n^{\prime}},\left\{w^{\prime}\right\}(x)\right]$ are generalizations (to interacting theories) of Wick's normal product, : $M_{n^{\prime},\left\{w^{\prime}\right\}}(x):_{C_{0}^{\Lambda_{0}}}$, because due to (4.10), (4.11) we find that

$$
L_{(0,0), 1}^{0, \Lambda_{0},}\left(N_{D}^{c}\left[M_{n^{\prime},\left\{w^{\prime}\right.}\right]\right)=-\int d^{4} x \chi(x) \cdot M_{n^{\prime},\left\{w^{\prime}\right\}}(x) .
$$

Returning to the discussion of how to apply Theorem 8 we require now that $D_{0}<D_{1}$ (cf. (4.3)); we choose a pair $\left(n^{\prime},\left\{w^{\prime}\right\}\right)$ with $n^{\prime} \geqq 1$ and $n^{\prime}+\left|w^{\prime}\right| \leqq D_{0}$ and set

$$
\begin{gathered}
B^{(0)}:=B_{D_{0},\left(n^{\prime},\left\{w^{\prime}\right\}\right)}, \\
\left\{B^{(j)}: 1 \leqq j \leqq N\right\}:=\left\{B_{D_{1},(n,\{w\})}: 1 \leqq n, n+|w| \leqq D_{1}\right\} .
\end{gathered}
$$


With this definition and (4.10) we easily see that the conditions (4.6) and (4.7) are fulfilled. Therefore there exist the linear relations (4.8) among the Zimmermann normal products $N_{D_{0}}^{c}[\cdots(x)]$ and $N_{D_{1}}^{c}[\cdots(x)]$, a priori at $x=0$ but after translation obviously at any $x$. Because of the very simple renormalization conditions (4.10) it is not difficult to calculate, from (4.9), also the coefficients $c_{r}^{(j)}, 1 \leqq j \leqq N$. We summarize our findings in the following proposition.

Proposition 9. The Zimmermann normal products $N_{D}^{c}$ obey the Zimmermann identities

$$
\begin{aligned}
& \mathscr{L}_{r, 1 ; n}^{\Lambda, \Lambda_{0}}\left(N_{D_{0}}^{c}\left[M_{n^{\prime},\left\{w^{\prime}\right\}}(x)\right] ; \ldots\right) \\
& =\sum_{\substack{r^{\prime}+r^{\prime \prime}=r \\
n^{\prime \prime},\left\{w^{\prime \prime}\right\}: n^{\prime \prime} \geqq 1, n^{\prime \prime}+\left|w^{\prime \prime}\right| \leqq D_{1}}} c_{r^{\prime}}^{\left(n^{\prime \prime},\left\{w^{\prime \prime}\right\}\right)} \cdot \mathscr{L}_{r^{\prime \prime}, 1 ; n}^{\Lambda, \Lambda_{0}}\left(N_{D_{1}}^{c}\left[M_{n^{\prime \prime},\left\{w^{\prime \prime}\right\}}(x)\right] ; \ldots\right),
\end{aligned}
$$

where $1 \leqq n^{\prime}+\left|w^{\prime}\right| \leqq D_{0}<D_{1}$ and

$$
c_{r^{\prime}}^{\left(n^{\prime \prime},\left\{w^{\prime \prime}\right\}\right)}=-\left(\mathscr{N}_{\left\{w^{\prime \prime}\right\}}\right)^{-1} \cdot \partial_{p}^{w^{\prime \prime}} \mathscr{L}_{r^{\prime}, 1 ; n^{\prime \prime}}^{0, \Lambda_{0}}\left(N_{D_{0}}^{c}\left[M_{n^{\prime},\left\{w^{\prime}\right\}}(0)\right] ; 0\right) .
$$

The explicit formula (4.15), together with (4.10) and (2.37), shows that for $n^{\prime \prime}+$ $\left|w^{\prime \prime}\right| \leqq D_{0}$

$$
c_{r^{\prime}}^{\left(n^{\prime \prime},\left\{w^{\prime \prime}\right\}\right)}=\delta_{r^{\prime},(0,0)} \cdot \delta_{n^{\prime \prime}, n^{\prime}} \cdot \delta_{\left\{w^{\prime \prime}\right\},\left\{w^{\prime}\right\}} \cdot
$$

In (4.14) we now take $\Lambda_{0} \rightarrow \infty, \Lambda \rightarrow 0$, multiply by $g^{r}$, sum over $r$, identify the perturbation series of the full Green functions with one insertion with the composite operators themselves and use (4.16) to arrive at

$$
\begin{aligned}
& N_{D_{0}}^{c}\left[M_{n^{\prime},\left\{w^{\prime}\right\}}(x)\right]=N_{D_{1}}^{c}\left[M_{n^{\prime},\left\{w^{\prime}\right\}}(x)\right] \\
& +\sum_{\substack{\left.n^{\prime \prime} \\
D_{0}<n^{\prime \prime}+\mid w^{\prime \prime}\right\}} \leqq D_{1}} N_{D_{1}}^{c}\left[M_{n^{\prime \prime},\left\{w^{\prime \prime}\right\}}(x)\right] \cdot c^{\left(n^{\prime \prime},\left\{w^{\prime \prime}\right\}\right)},
\end{aligned}
$$

which corresponds to the conventional form of the standard Zimmermann identities $[1,2]$.

4.3. As a second application of Theorem 8 we will discuss some aspects of the standard Zimmermann normal products $N_{D}^{p}[\cdots]$.

For $D \geqq n^{\prime}+\left|w^{\prime}\right|$ let $B_{D,\left(n^{\prime},\left\{w^{\prime}\right\}\right)}^{p}$ be the unique polynomial such that the proper Green functions with one insertion of $B_{D,\left(n^{\prime},\left\{w^{\prime}\right\}\right)}^{p}(0)$ respect the renormalization conditions (2.37), (4.10) which before have been imposed on the connected amputated Green functions. We now define

$$
\mathscr{L}_{r, 1 ; n}^{\Lambda, \Lambda_{0}}\left(N_{D}^{p}\left[M_{n^{\prime},\left\{w^{\prime}\right\}}(x)\right] ; \ldots\right):=\mathscr{L}_{r, 1 ; n}^{\Lambda, \Lambda_{0}}\left(B_{D,\left(n^{\prime},\left\{w^{\prime}\right\}\right)}^{p}(x) ; \ldots\right) .
$$

Restrict the allowed renormalization conditions $(2.30)$ by the requirement $a_{r}^{(1) R}=0, \forall r$; this corresponds precisely to Zimmermann's prescription concerning the tadpoles [2]. Standard considerations relying on (3.2), and on (3.11) for $t=0$, and on the renormalization conditions on the proper functions with one insertion show that the relevant and marginal parts $(2.37)$ of $\mathscr{L}_{r, 1 ; n}^{0, \Lambda_{0}}\left(N_{D}^{p}\left[M_{n^{\prime},\left\{w^{\prime}\right\}}(0)\right] ; \ldots\right)$ are indeed finite and only weakly $\Lambda_{0}$-dependent. Now Theorems 3 and (a slight variant of) 4 can be applied to prove that $\lim _{\Lambda_{0} \rightarrow \infty} \mathscr{L}_{r, 1 ; n}^{0, \Lambda_{0}}\left(N_{D}^{p}\left[M_{n^{\prime},\left\{w^{\prime}\right\}}(0)\right] ; \ldots\right)$ exists. Moreover one readily verifies that

$$
\mathscr{L}_{r=0,1 ; n}^{0, \Lambda_{0}}\left(N_{D}^{p}\left[M_{n^{\prime},\left\{w^{\prime}\right\}}(x)\right] ; \ldots\right)=\mathscr{L}_{r=0,1 ; n}^{0, \Lambda_{0}}\left(N_{D}^{c}\left[M_{n^{\prime},\left\{w^{\prime}\right\}}(x)\right] ; \ldots\right)^{\prime},
$$


for all $n \geqq 0$, and that (using the above restriction on (2.30)) $\mathscr{L}_{r, 1 ; 0}^{0, \Lambda_{0}}\left(N_{D}^{p}\left[M_{n^{\prime},\left\{w^{\prime}\right\}}(x)\right]\right)=0$, for all $|r| \geqq 0$. So, in the $\Phi_{4}^{4}$ theory with the BPHZ condition $a_{r}^{(1) R}=0, \forall r$, we can use Theorem 8 to show that for example:

1. There are coefficients $c_{r^{\prime}}^{\left(n^{\prime \prime},\left\{w^{\prime \prime}\right\}\right)}$, which in general are different from those in (4.15), such that the Zimmermann identities (4.14) hold also for the $N_{D}^{p}[\cdots]$ (proof: simply put $D_{0}<D_{1}$ and replace $B \ldots \mapsto B_{\ldots}^{p}$ in (4.13)). Making use of the normalization conditions on the $N_{D}^{p}[\cdots]$ one can compute these coefficients; one recovers the result $[1,2]$ that $\left.c_{r^{\prime}}^{\left(n^{\prime \prime}\right.},\left\{w^{\prime \prime}\right\}\right)$ is given by the analog for the proper Green functions of the r.h.s. of (4.15). In this way one finds the standard Zimmermann identities (4.17) for the $N_{D}^{p}[\cdots]$.

2. $N_{D}^{p}[\cdots]$ is a linear combination of the $N_{D}^{c}[\cdots]$ (proof: put $D=D_{0}=D_{1}$ and $B^{(0)}:=B_{D,\left(n^{\prime},\left\{w^{\prime}\right\}\right)}^{p}$ in (4.13)); and vice versa. In this sense these two types of normal products are equivalent.

4.4. One of the virtues of the normal products $N_{D}^{p, c}[\cdots]$ is that they commute with derivatives and that $N_{D}^{p, c}\left[M_{n^{\prime},\left\{w^{\prime}\right\}}(x)\right]$ behaves just as $M_{n^{\prime},\left\{w^{\prime}\right\}}(x)$ if it is subjected to $O(4)$ transformations. The formula which expresses the former property for $N_{D}^{p}$ is known as Lowenstein's rule [4]; the latter property for $N_{D}^{p}$ is certainly well-known as well but we were not able to find a corresponding formula in the literature. In the remainder of this section we will give a proof of these two properties for $N_{D}^{c}$, the main technical tool being Lemma 6 .

Using (2.22) and (4.11) it can be shown without difficulty that

$$
\begin{aligned}
& \mathscr{L}_{r, 1 ; n}^{\Lambda, \Lambda_{0}}\left(N_{D}^{c}\left[M_{n^{\prime},\left\{w^{\prime}\right\}}(x)\right] ; p_{1}, \ldots, p_{n}\right) \\
& \quad=\left\{\begin{array}{l}
e^{i\left(\sum_{\jmath=1}^{n} p_{J}\right) \cdot x} \cdot \mathscr{L}_{r, 1 ; n}^{\Lambda, \Lambda_{0}}\left(N_{D}^{c}\left[M_{n^{\prime},\left\{w^{\prime}\right\}}(0)\right] ; p_{1}, \ldots, p_{n}\right), \quad n \geqq 1 \\
\mathscr{L}_{r, 1 ; 0}^{\Lambda, \Lambda_{0}}\left(N_{D}^{c}\left[M_{n^{\prime},\left\{w^{\prime}\right\}}(0)\right]\right), \quad n=0 .
\end{array}\right.
\end{aligned}
$$

As usual the Green function $\left.\mathscr{L}_{r, 1 ; n}^{\Lambda, \Lambda_{0}} \partial_{\mu} N_{D}^{c}\left[M_{n^{\prime},\left\{w^{\prime}\right\}}(x)\right] ; p_{1}, \ldots, p_{n}\right)$ with one insertion of the operator $\partial_{\mu} N_{D}^{c}[\cdots] \equiv \partial_{x_{\mu}} N_{D}^{c}[\cdots]$ at $x$ is defined as

$$
\mathscr{L}_{r, 1 ; n}^{\Lambda, \Lambda_{0}}\left(\partial_{\mu} N_{D}^{c}\left[M_{n^{\prime},\left\{w^{\prime}\right\}}(x)\right] ; p_{1}, \ldots, p_{n}\right):=\partial_{x_{\mu}} \mathscr{L}_{r, 1 ; n}^{\Lambda, \Lambda_{0}}\left(N_{D}^{c}\left[M_{n^{\prime},\left\{w^{\prime}\right\}}(x)\right] ; p_{1}, \ldots, p_{n}\right) .
$$

Putting $x=0$ we thus find that

$$
\begin{aligned}
& \mathscr{L}_{r, 1 ; n}^{\Lambda, \Lambda_{0}}\left(\partial_{\mu} N_{D}^{c}\left[M_{n^{\prime},\left\{w^{\prime}\right\}}(0)\right] ; p_{1}, \ldots, p_{n}\right) \\
& \quad=\left\{\begin{array}{l}
\left(\sum_{j=1}^{n} i p_{j, \mu}\right) \cdot \mathscr{L}_{r, 1 ; n}^{\Lambda, \Lambda_{0}}\left(N_{D}^{c}\left[M_{n^{\prime},\left\{w^{\prime}\right\}}(0)\right] ; p_{1}, \ldots, p_{n}\right), n \geqq 1 . \\
0, \quad n=0 .
\end{array}\right.
\end{aligned}
$$

Next, we extend the definition of $N_{D}^{c}[\cdots]$ from monomials to polynomials by linearity, i.e.

$$
N_{D}^{c}\left[\sum_{n^{\prime}+\left|w^{\prime}\right| \leqq D} c_{n^{\prime},\left\{w^{\prime}\right\}} \cdot M_{n^{\prime},\left\{w^{\prime}\right\}}(x)\right]:=\sum_{n^{\prime}+\left|w^{\prime}\right| \leqq D} c_{n^{\prime},\left\{w^{\prime}\right\}} \cdot N_{D}^{c}\left[M_{n^{\prime},\left\{w^{\prime}\right\}}(x)\right] .
$$

Thus for $D \geqq n^{\prime}+\left|w^{\prime}\right|$ the r.h.s. of

$$
\begin{aligned}
\mathscr{F}_{r, 1 ; n}^{\Lambda, \Lambda_{0}}(\ldots):= & \mathscr{L}_{r, 1 ; n}^{\Lambda, \Lambda_{0}}\left(N_{D+1}^{c}\left[\partial_{\mu} M_{n^{\prime},\left\{w^{\prime}\right\}}(0)\right] ; \ldots\right) \\
& -\mathscr{L}_{r, 1 ; n}^{\Lambda, \Lambda_{0}}\left(\partial_{\mu} N_{D}^{c}\left[M_{n^{\prime},\left\{w^{\prime}\right.}^{c}(0)\right] ; \ldots\right)
\end{aligned}
$$


is well-defined. It is straightforward to check that $\mathscr{F}_{r, 1 ; n}^{\Lambda, \Lambda_{0}}$ satisfies (4.1); moreover, putting $d:=D+1$ and using e.g. (4.12) it is not difficult to verify that $\mathscr{F}_{r, 1 ; n} \Lambda_{0}$ fulfills also a)-d) of Lemma 6. Therefore we have proved

Proposition 10. (Lowenstein's rule). If $D \geqq n^{\prime}+\left|w^{\prime}\right|$ then

$$
\partial_{\mu} N_{D}^{c}\left[M_{n^{\prime},\left\{w^{\prime}\right\}}(x)\right]=N_{D+1}^{c}\left[\partial_{\mu} M_{n^{\prime},\left\{w^{\prime}\right\}}(x)\right] .
$$

4.5. Now let $A \in O(4)$ and $p_{j}^{\prime}:=A p_{j}$, for $1 \leqq j \leqq n^{\prime}$. There are coefficients $T_{u}^{w}=T_{u}^{w}(A)$ so that

$$
\begin{aligned}
\partial_{p}^{w} & =\sum_{u:\left|u_{j}\right|=\left|w_{j}\right|, 1 \leqq j \leqq n^{\prime}} T_{u}^{w} \cdot \partial_{p^{\prime}}^{u} \\
& =\sum_{\{u\}} \kappa_{\{u\}}\left(\sum_{\pi \in S_{n^{\prime}}} T_{u^{\pi}}^{w} \cdot \prod_{j=1}^{n^{\prime}} \delta_{\left|w_{j}\right|,\left|u_{j}^{\pi}\right|} \cdot \partial_{p^{\prime}}^{u^{\pi}}\right),
\end{aligned}
$$

where $w=\left(w_{1}, \ldots, w_{n^{\prime}}\right), u=\left(u_{1}, \ldots, u_{n^{\prime}}\right), u^{\pi}=\left(u_{\pi(1)}, \ldots, u_{\pi\left(n^{\prime}\right)}\right)$, and where $\kappa_{\{u\}}$ is (the inverse of) some combinatorial factor. We define a symmetrized version of $T_{w^{\prime}}^{w}$ by

$$
\begin{aligned}
\beta_{\left\{w^{\prime}\right\}}^{\{w\}} & :=\frac{1}{n^{\prime} !} \sum_{\pi \in S_{n^{\prime}}}\left(\sum_{u:\left|u_{j}\right|=\left|w_{J}^{\pi}\right|, \forall j} T_{u}^{w^{\pi}} \cdot \delta_{\{u\},\left\{w^{\prime}\right\}}\right) \cdot \frac{\mathscr{N}_{\left\{w^{\prime}\right\}}}{\mathscr{N}_{\{w\}}} \\
& =\frac{1}{n^{\prime} !} \sum_{\pi, \pi^{\prime} \in S_{n^{\prime}}}\left(T_{w^{\prime \pi^{\prime}}}^{w^{\pi}} \prod_{j=1}^{n^{\prime}} \delta_{\left|w_{j}^{\pi}\right|,\left|w_{j}^{\prime \pi^{\prime} \mid}\right|}\right) \cdot \kappa_{\left\{w^{\prime}\right\}} \cdot \frac{\mathcal{N}_{\left\{w^{\prime}\right\}}}{\mathcal{N}_{\{w\}}} ;
\end{aligned}
$$

obviously the r.h.s. of (4.23) depends on $w, w^{\prime}$ only through $\{w\},\left\{w^{\prime}\right\}$ thus justifying the notation on the 1.h.s. It is also evident that $\beta_{\left\{w^{\prime}\right\}}^{\{w\}}=0$ if $|w| \neq\left|w^{\prime}\right|$. We assume that $D \geqq n^{\prime}+\left|w^{\prime}\right|$ and put

$$
\begin{aligned}
\mathscr{F}_{r, 1 ; n}^{\Lambda, \Lambda_{0}}\left(p_{1}, \ldots, p_{n}\right):= & \sum_{\left\{w^{\prime \prime}\right\}} \beta_{\left\{w^{\prime}\right\}}^{\left\{w^{\prime \prime}\right\}} \cdot \mathscr{L}_{r, 1 ; n}^{\Lambda, \Lambda_{0}}\left(N_{D}^{c}\left[M_{n^{\prime},\left\{w^{\prime \prime}\right\}}(0)\right] ; p_{1}, \ldots, p_{n}\right) \\
& -\mathscr{L}_{r, 1 ; n}^{\Lambda, \Lambda_{0}}\left(N_{D}^{c}\left[M_{n^{\prime},\left\{w^{\prime}\right\}}(0)\right] ; A p_{1}, \ldots, A p_{n}\right) .
\end{aligned}
$$

Using the fact that the $\Phi_{4}^{4}$ Green functions $\mathscr{L}_{r, 0 ; n}^{\Lambda, \Lambda_{0}}$ as well as $\partial_{\Lambda} R(\Lambda, \cdot)$ are invariant under $O(4)$ it is an easy task to check that $\mathscr{F}_{r, 1 ; n} \Lambda, \Lambda_{0}$ again obeys the differential flow equation (4.1). With $d:=D$ and (4.23) also the conditions a)-d) of Lemma 6 are fulfilled, hence $\mathscr{F}^{\Lambda, \Lambda}, \Lambda_{0} \equiv 0$. If we set $\Lambda=0, r=0$ in (4.24) and make use of (4.12) we see that the $\beta_{\left\{w^{\prime}\right\}}^{\left\{w^{\prime \prime}\right\}}$ are precisely the coefficients which describe $O(4)$ transformations of $M_{n^{\prime},\left\{w^{\prime}\right\}}$. As a result we have found

Proposition 11. Let $\beta_{\left\{w^{\prime}\right\}}^{\{w\}}$ be defined as in (4.23). Then the $O(4)$ covariance of the normal product $N_{D}^{c}\left[M_{n^{\prime}},\left\{w^{\prime}\right\}(x)\right]$ is displayed by

$$
\begin{aligned}
\mathscr{L}_{r, 1 ; n}^{\Lambda, \Lambda_{0}}\left(N_{D}^{c}\left[M_{n^{\prime},\left\{w^{\prime}\right\}}(0)\right] ; A p_{1}, \ldots, A p_{n}\right) \\
\quad=\sum_{\left\{w^{\prime \prime}\right\}} \beta_{\left\{w^{\prime}\right\}}^{\left\{w^{\prime \prime}\right\}} \cdot \mathscr{L}_{r, 1 ; n}^{\Lambda, \Lambda_{0}}\left(N_{D}^{c}\left[M_{n^{\prime},\left\{w^{\prime \prime}\right\}}(0)\right] ; p_{1}, \ldots, p_{n}\right),
\end{aligned}
$$

which holds for all $A \in O(4)$.

Let us remark that the same simple methods could of course also be used to prove the analogous results for the standard Zimmermann normal products $N_{D}^{p}$, e.g., but the explicit computations would become somewhat more involved. 


\section{Appendix}

Here we outline the essential steps leading to the proof of Theorem 5. Define the fps $G^{\Lambda, \Lambda_{0}}$ by

$$
G^{\Lambda, \Lambda_{0}}:=-\log \left(\left.e^{\Delta\left(\Lambda, \Lambda_{0}\right)} e^{\left.-G^{\Lambda_{0}, \Lambda_{0}}\right)}\right|_{\text {field-dep. }},\right.
$$

where for all $\theta$ with $\theta \geqq \theta_{\min }$ (actually, at this point, we can admit any $\theta>0$ )

$$
G^{\Lambda_{0}, \Lambda_{0}}:=L^{\Lambda_{0}, \Lambda_{0}}-\mu \cdot \partial_{\theta} L^{\Lambda_{0}, \Lambda_{0}}
$$

and where $\mu$ is a formal variable; $L^{\Lambda_{0}, \Lambda_{0}}$ is given in (2.5). Expand

$$
G^{\Lambda, \Lambda_{0}}=G^{(0) \Lambda, \Lambda_{0}}+\mu \cdot G^{(1) \Lambda, \Lambda_{0}}+O\left(\mu^{2}\right)
$$

and notice that due to (A.1), (A.2)

$$
G^{(0) \Lambda, \Lambda_{0}} \equiv L^{\Lambda, \Lambda_{0}} .
$$

Acting with $\partial_{\theta}$ on (2.9) we obtain after a simple calculation

$$
\begin{aligned}
\partial_{\theta} L^{\Lambda, \Lambda_{0}}= & \left.\left(\partial_{\theta} \Delta\left(\Lambda, \Lambda_{0}\right)\right) L^{\Lambda, \Lambda_{0}}\right|_{\text {field-dep. }} \\
& -\left.\frac{1}{2}\left\langle\delta_{\phi} L^{\Lambda, \Lambda_{0}},\left(\partial_{\theta} C_{\Lambda}^{\Lambda_{0}}\right) \delta_{\phi} L^{\Lambda, \Lambda_{0}}\right\rangle\right|_{\text {field-dep. }} \\
& -G^{(1) \Lambda, \Lambda_{0}} .
\end{aligned}
$$

Expanding (A.5) as a fps in $g_{1}, g_{2}$ and $\lambda$ and putting $\Lambda=0$ we arrive at

$$
\begin{aligned}
\partial_{\theta} \mathscr{L}_{r, t ; n}^{0, \Lambda_{0}}\left(p_{1}, \ldots, p_{n+t-1}\right)= & \text { (r.h.s. of }(2.23))\left.\right|_{\partial_{A} R(\Lambda, \cdot) \mapsto-\partial_{\theta} R\left(\Lambda_{0}, \cdot\right) ; \Lambda=0} \\
& -\mathscr{G}_{r, t ; n}^{(1) 0, \Lambda_{0}}\left(p_{1}, \ldots, p_{n+t-1}\right),
\end{aligned}
$$

for $t=0,1$ and at least for all $\theta$ with $\theta \geqq \theta_{\min }$.

Let us bound the first term on the r.h.s. of (A.6). We begin by recalling that $\mathscr{L}_{r, t ; n}^{0, \Lambda_{0}}$ is given by a finite sum of contributions stemming from connected amputated Feynman diagrams, i.e.

$$
\begin{aligned}
\mathscr{L}_{r, t ; n}^{0, \Lambda_{0}}\left(p_{1}, \ldots, p_{n+t-1}\right)= & \left.\sum_{\text {finite }} \text { (combinatorial factor) }\right) \cdot \int \frac{d^{4} q_{1}}{(2 \pi)^{4}} \cdots \frac{d^{4} q_{L}}{(2 \pi)^{4}} \\
& \cdot\left(\prod_{l} \frac{R\left(\Lambda_{0}, Q_{l}\right)}{Q_{l}^{2}+m^{2}}\right) \cdot\left(\prod_{v} \mathscr{L}_{r_{v}, t_{v} ; n_{v}}^{\Lambda_{0}, \Lambda_{0}}(\vec{Q}, \vec{p})\right),
\end{aligned}
$$

where $\prod_{l}, \prod_{v}$ extends over all lines resp. vertices of the Feynman diagram under consideration, $\left\{q_{1}, \ldots, q_{L}\right\} \subset\left\{Q_{1}, Q_{2}, \ldots\right\}$, and the momenta $Q$ which obey $Q \in$ $\left\{Q_{1}, Q_{2}, \ldots\right\} \backslash\left\{q_{1}, \ldots, q_{L}\right\}$ are linear combinations of $q_{1}, \ldots, q_{L}, p_{1}, \ldots, p_{n+t-1}$. Thus, for $0 \leqq|w| \leqq \theta-1$ and $\left|p_{i}\right| \leqq 2 \Lambda_{0}$ we find

$$
\begin{aligned}
\left|\partial_{p}^{w} \mathscr{L}_{r, t ; n}^{0, \Lambda_{0}}\left(p_{1}, \ldots, p_{n+t-1}\right)\right| \leqq & \sum_{\text {finite }} \text { const } \cdot \int_{0}^{2 \Lambda_{0}} \frac{d^{4} q_{1}}{(2 \pi)^{4}} \cdots \frac{d^{4} q_{L}}{(2 \pi)^{4}} \\
& \times \max _{\left|Q_{J}\right| \leqq 2 \Lambda_{0}} \prod_{v}\left|\partial_{p, Q}^{w_{v}} \mathscr{L}_{r_{v}, t_{v} ; n_{v}}^{\Lambda_{0}, \Lambda_{0}}(\vec{Q}, \vec{p})\right|
\end{aligned}
$$




$$
\begin{aligned}
& \leqq \sum_{\text {finite }} \operatorname{const} \cdot\left(\Lambda_{0}\right)^{4 L} \cdot \prod_{v}\left\|\partial^{\left|w_{v}\right|} \mathscr{L}_{r_{v}, t_{v} ; n_{v}}^{\Lambda_{0}, \Lambda_{0}}\right\|_{\left(2 \Lambda_{0}, 0\right)} \\
& \leqq P\left(\frac{\Lambda_{0}}{\Lambda_{1}}\right)
\end{aligned}
$$

for $t=0,1$, where we have used (3.2) and (3.3). Let us remark that an alternative way (which, moreover, would be more in the spirit of this paper) to derive the bound (A.8) would consist of a repetition of the proof of Theorem 3 such that $\left\|\partial^{z} \mathscr{L}_{r, t ; n}^{\Lambda, \Lambda_{0}}\right\|_{\left(2 \Lambda, 2 \Lambda_{0}\right)} \leqq P\left(\frac{\Lambda_{0}}{\Lambda_{1}}\right)$ can be established. Anyway, the inequality (A.8) provides the estimates we sought for the $\mathscr{L}$ 's appearing in the first term on the r.h.s. of (A.6). Using the fact that for all $w$ and $\Lambda_{0} \geqq \Lambda_{0, \min }>0$,

$$
\left|\partial_{p}^{w} \partial_{\theta} R\left(\Lambda_{0}, p\right)\right| \leqq \mathrm{const} \cdot \Lambda_{0}^{\theta-|w|} \cdot \log \left(\frac{\Lambda_{0}}{m}\right) \cdot e^{-\left(\Lambda_{0} / m\right)^{\theta}} \leqq \text { const } \cdot e^{-\left(\Lambda_{0} / m\right)^{2}}
$$

(because $\theta_{\min }>2$ ) together with (A.8) we obtain for $0 \leqq|w| \leqq \theta-1$ and $\left|p_{i}\right| \leqq 2 \Lambda_{0}, 1 \leqq i \leqq n+t-1$ :

$$
\mid \partial_{p}^{w}\left(\text { first term on r.h.s. of }(\text { A.6) }) \mid \leqq \text { const } \cdot e^{-\Lambda_{0} / m}\right. \text {. }
$$

So we are left with verifying that the same bounds hold for the second term on the r.h.s. of (A.6).

Because the renormalization conditions for the $\mathscr{L}_{\mathrm{S}}$ are $\theta$-independent we have

$$
\partial_{\theta} \partial_{p}^{w} \mathscr{L}_{r, t ; n}^{0, \Lambda_{0}}(0)=0, \quad \text { if } n+|w| \leqq 4(1-t)+D \cdot t ;
$$

hence, combining (A.6), (A.10) and (A.11) we see that

$$
\left|\partial_{p}^{w} \mathscr{G}_{r, t ; n}^{(1) 0, \Lambda_{0}}(0)\right| \leqq \text { const } \cdot e^{-\Lambda_{0} / m}, \quad \text { if } n+|w| \leqq 4(1-t)+D \cdot t .
$$

Referring to (A.2), (A.3) we note that $G^{(1) \Lambda_{0}, \Lambda_{0}}=-\partial_{\theta} L^{\Lambda_{0}, \Lambda_{0}}$, thus

$$
\partial_{p}^{w} \mathscr{G}_{r, t ; n}^{(1) \Lambda_{0}, \Lambda_{0}} \equiv 0, \quad \text { if } n+|w| \geqq 4(1-t)+D \cdot t+1 .
$$

Acting with $\partial_{\Lambda}$ on (A.1) we can derive a linear differential flow equation for $\mathscr{G}_{r, t ; n}^{(1) \Lambda, \Lambda_{0}}$, $t=0,1$. Using the bc (A.12), (A.13), the bounds (3.2), (3.3) and techniques which are similar to those employed in the proof of Theorem 3 we can show that (among others)

$$
\left\|\mathscr{G}_{r, t ; n}^{(1) 0, \Lambda_{0}}\right\|_{(0, \eta)} \leqq \text { const } \cdot e^{-\Lambda_{0} / m} .
$$

(A.6) with (A.10) and (A.14) yields Theorem 5.

\section{References}

1. Zimmermann, W.: Local operator products and renormalization in quantum field theory. In: Lectures on Elementary Particles and Quantum Field Theory; Brandeis Summer Institute 1970, Deser, S., Grisaru, M., Pendleton, H. (eds.). Cambridge, MA: MIT Press 1970

2. Zimmermann, W.: Ann. Phys. 77, 536 (1973)

3. Zimmermann, W.: Ann. Phys. 77, 570 (1973)

4. Lowenstein, J.: Phys. Rev. D4, 2281 (1971)

5. Lowenstein, J.: Commun. Math. Phys. 24, 1 (1971)

6. Schroer, B.: Lett. Nuovo Cimento 2, 867 (1971)

7. Gomes, M., Lowenstein, J.: Nucl. Phys. B45, 252 (1972) 
8. Lowenstein, J., Schroer, B.: Phys. Rev. D6, 1553 (1972)

9. Lowenstein, J.: BPHZ Renormalization. In: Renormalization theory; Erice Advanced Study Institute 1975, Velo, G., Wightman, A. (eds.), Amsterdam: D. Reidel 1976

10. Lowenstein, J.: 1972 Maryland Lectures, Univ. of Maryland Technical Report 73-068

11. Gallavotti, G., Nicolò, F.: Commun. Math. Phys. 100, 545 (1985); Gallavotti, G., Nicolò, F.: Commun. Math. Phys. 101, 247 (1986)

12. Feldman, J., Hurd, T., Rosen, L., Wright, J.: QED: A proof of renormalizability, Lecture Notes in Physics vol. 312, Berlin, Heidelberg, New York: Springer 1988

13. Hurd, T.: Commun. Math. Phys. 125, 515 (1989)

14. Rosen, L., Wright, J.: Commun. Math. Phys. 134, 433 (1990)

15. Polchinski, J.: Nucl. Phys. B231, 269 (1984)

16. Keller, G., Kopper, C., Salmhofer, M.: Perturbative Renormalization and Effective Lagrangians in $\Phi_{4}^{4}$. MPI preprint MPI-PAE/PTh 65/90, Helv. Phys. Acta, to appear

17. Keller, G., Kopper, C.: Perturbative Renormalization of QED via Flow Equations. Phys. Lett. B, to appear; Keller, G., Kopper, C.: preprint in preparation

18. Hurd, T.: Commun. Math. Phys. 124, 153 (1989)

19. Keller, G., Kopper, C.: preprint in preparation

Communicated by J. Fröhlich 
